\title{
Sensitivity of in-plane strain measurement to calibration parameter for out-of-plane specimen rotations
}

\author{
Farzana Yasmeen ${ }^{1}$, Ruben Balcaen ${ }^{4}$, Michael A Sutton ${ }^{2}$, Dimitri Debruyne ${ }^{5}$, Sreehari Rajan ${ }^{1}$ and Hubert W. Schreier ${ }^{3}$ \\ ${ }^{1} \mathrm{PhD}$ Student and ${ }^{2}$ Professor, Department of Mechanical Engineering, University of South Carolina \\ ${ }^{3}$ Correlated Solutions Incorporated, 121 Dutchman Blvd, Irmo, SC 29063 \\ ${ }^{4} \mathrm{PhD}$ Student and ${ }^{5}$ Professor, Department of Materials Engineering, University of Leuven, Belgium
}

\begin{abstract}
In practice, out-of-plane motions usually are not avoidable during experiments. Since 2D-DIC measurements are vulnerable to parasitic deformations due to out-of-plane specimen motions, three-dimensional digital image correlation (StereoDIC or 3DDIC) oftentimes is employed. The StereoDIC method is known to be capable of accurate deformation measurements for specimens subjected to general three-dimensional motions, including out-of-plane rotations and displacements. As a result, there has been limited study of the deformation measurements obtained when using StereoDIC to measure the displacement and strain fields for a specimen subjected only to out-of-plane rotation. To assess the accuracy of strain measurements obtained using stereovision systems and StereoDIC when a specimen undergoes appreciable out of plane rotation, rigid body out-of-
\end{abstract} plane rotation experiments are performed in the range $-40^{\circ} \leq \theta \leq 40^{\circ}$ using a two-camera stereovision system. Results indicate that (a) for what would normally be considered "small angle" calibration processes, the measured normal strain in the foreshortened specimen direction due to specimen rotation increases in a non-linear manner with rotation angle, with measurement errors exceeding $\pm 1400 \mu \varepsilon$ and (b) for what would normally be considered "large angle" calibration processes, the magnitude of the errors in the strain are reduced to $\pm 300 \mu \varepsilon$. To theoretically assess the effect of calibration parameters on the measurements, two separate analyses are performed. First, theoretical strains due to out-of-plane rigid body rotation are determined using a pinhole camera model to project a series of three-dimensional object points into the image plane using large angle calibration parameters and then re-project the corresponding sensor plane coordinates back into the plane using small angle calibration parameters. Secondly, the entire imaging process is also simulated in order to remove experimental error sources and to further validate the theory. Results from both approaches confirmed the same strain error trends as the experimental strain measurements, providing confidence that the source of the errors is the calibration process. Finally, variance based sensitivity analyses show that inaccuracy in the calibrated stereo angle parameter is the most significant factor affecting the accuracy of the measured strain.

KEYWORDS: Stereo digital image correlation, rigid body out-of-plane rotation, stereo calibration, measurement error 


\section{INTRODUCTION}

Digital image correlation (DIC) is a non-contacting measurement technique capable of measuring full field deformation on a specimen's surface [1]. DIC was first originated and devised for planar surfaces undergoing in-plane deformations in the early 1980s [2] and was extended to general deformations of curved or planar surfaces in the early 1990s [3]-[5]. Since that time, the DIC technique has grown into one of the most popular measurement method worldwide. In principle, the method known as 2D-DIC gives accurate results when a nominally planar specimen is subjected to in-plane mechanical loading. In such cases, the planar surfaces experience in plane deformations. If a single viewing camera is placed perpendicular to the specimen, then the measured deformations would reflect the true deformations under ideal conditions. However, as shown previously [6], any out-of-plane displacement or out-of-plane rotation of the specimen will induce in-plane displacement gradients that deleteriously affect the measured strains. Designated 3D-DIC or StereoDIC, a typical stereovision system employs two or more cameras to record digital images of a common object region from two or more viewpoints. Using DIC to perform cross-camera subset matching, a calibrated stereovision system theoretically can obtain the true, threedimensional (3D) position of each point on a non-planar object. StereoDIC is capable of measuring all three displacements and the entire motion of the specimen, thereby conceptually eliminating the deleterious effects of out-of-plane motion [6].

As demonstrated in previous work ([7], [8], [[9]) stereovision systems measure all three components of displacement simultaneously using at least two camera views. Calibration of the stereovision system typically is performed using a translated and rotated planar dot pattern with reasonably well-known spacing. In this work, the use of relatively small in-plane and out of plane rotations (e.g., rotations $\leq 10^{\circ}$ ) is designated small angle calibration (SAC). Conversely, the use of large in-plane and out-of-plane rotations (e.g. rotations up to $50^{\circ}$ ) is known as large angle calibration (LAC). To perform camera calibration, a large number of calibration methods have been proposed by computer vision researchers over the years. Among the linear calibration methods, the algorithm proposed by Ravn et al. [10] is attractive since it is simple to implement and only requires a planar calibration target that is easily manufactured. The resulting calibration process provides a direct approach for estimating camera parameters from a set of linear equations through a complex restructuring of unknowns into specific groupings. The procedure provides a theoretically sound approach for determining initial estimates for camera parameters in the absence of distortion and has been used for this purpose effectively in many practical applications. However, the linear calibration method does not permit the determination of the non-linear distortion parameters, requiring that the calibration points on the target are known with a high degree of accuracy. These limitations can be overcome using the bundle adjustment technique developed for photogrammetry applications. The bundle adjustment method permits the calibration of non-linear camera models while simultaneously determining the shape of the calibration object up to a scale factor [1]. Bundle adjustment 
is the process of jointly refining a set of initial camera and structure parameter estimates to determine the set of parameters that most accurately predict the locations of the observed points in a set of available images. In bundle adjustment it is assume that a total of $\mathrm{N}$ 3D points are seen in $\mathrm{M}$ views and the process is briefly described as follows. Defining a vector quantity using either bold $\xi$ or an overbar vector, $\vec{\xi}$, we let (a) $\vec{x}_{i j}$ be the projection of the $i^{t h}$ point onto image $j$, (b) $v_{i j}$ be the binary variables that equal 1 if point $i$ is visible in image $j$ and 0 otherwise, (c) each camera $j$ be parameterized by a vector $\vec{a}_{j}$ of "camera parameters" and (d) each 3D point by a vector $\vec{b}_{i}$. Then, the bundle adjustment process minimizes the total re-projection error with respect to all 3D points and camera parameters [12] as follows;

$$
\vec{\xi}=\min _{\vec{a}_{i} \vec{b}_{j}} \sum_{i=1}^{N} \sum_{j=1}^{M} v_{i j} d\left\{\vec{Q}\left(\vec{a}_{i}, \vec{b}_{j}\right), \vec{x}_{i j}\right\}^{2}
$$

Where $\mathbf{Q}\left(\mathrm{a}_{\mathrm{i}}, \mathrm{b}_{\mathrm{j}}\right)$ is the predicted projection of point $\mathrm{i}$ on image $\mathrm{j}$ and $d(\vec{x}, \vec{y})$ denotes the Euclidean distance between the image points represented by vectors $\vec{x}$ and $\vec{y}$. Clearly, bundle adjustment is by definition tolerant to missing image projections and minimizes a physically meaningful criterion in the image plane.

Since StereoDIC measures all three-dimensional motions simultaneously, the three-dimensional position of each object point is measured so that rigid body, out-of-plane motion of an object should not affect strain measurements. Put simply, StereoDIC is theoretically capable of extracting accurate, in-plane surface deformations, even when the object is undergoing relatively large, three-dimensional rigid body rotation and translation. However, there have been studies published [13] indicating that there is some effect of out-of-plane rotation on StereoDIC deformation (strain) measurements, where the predicted strains corrupt the measurements of interest as they are not due to the loading or environmental excitation. Since there are applications where large rotations may be present (e.g. large torsional deformation, outward bending of a beam and expansion of a balloon [14]), in the enclosed work the authors have focused on the effects of out-of-plane rotation on 3D-DIC deformation measurements. The theoretical stereo imaging equations using established pinhole models are presented in Section 2. Section 3 outlines sensitivity analysis theory that is employed when there are correlated input parameters. . Sections 4 and 5 present the out-of-plane rotation experimental considerations and the results and discussion, respectively. Section 6 describes analytic predictions based on two distinctly different calibration processes, designated as large angle calibration (LAC) and small angle calibration (SAC). Section 7 outlines the results using simulated images. Section 8 presents sensitivity analysis results. Finally Sections 9 and 10 provide a detailed discussion of the results and concluding remarks, respectively. 


\section{Pinhole Camera Stereo Imaging Theory}

A top view of a two-camera stereovision system is shown schematically in Figure 1. As shown in Figure 1, if a threedimensional point $\mathrm{O}$ is imaged by two separate cameras, then its location on each sensor plane can be determined using an appropriate imaging model. If we do not know the size of the object associated with point $\mathrm{O}$, and we only use the sensor position in one camera, then we cannot determine where along the corresponding ray the point is located. However, if two views are obtained and the perspective projection imaging model is employed to define the imaging process for each camera, then we can identify the location where rays $\mathrm{O}_{1}-\mathrm{O}$ and $\mathrm{O}_{2}-\mathrm{O}$ intersect and thereby determine the three-dimensional position, $\mathrm{O}$.

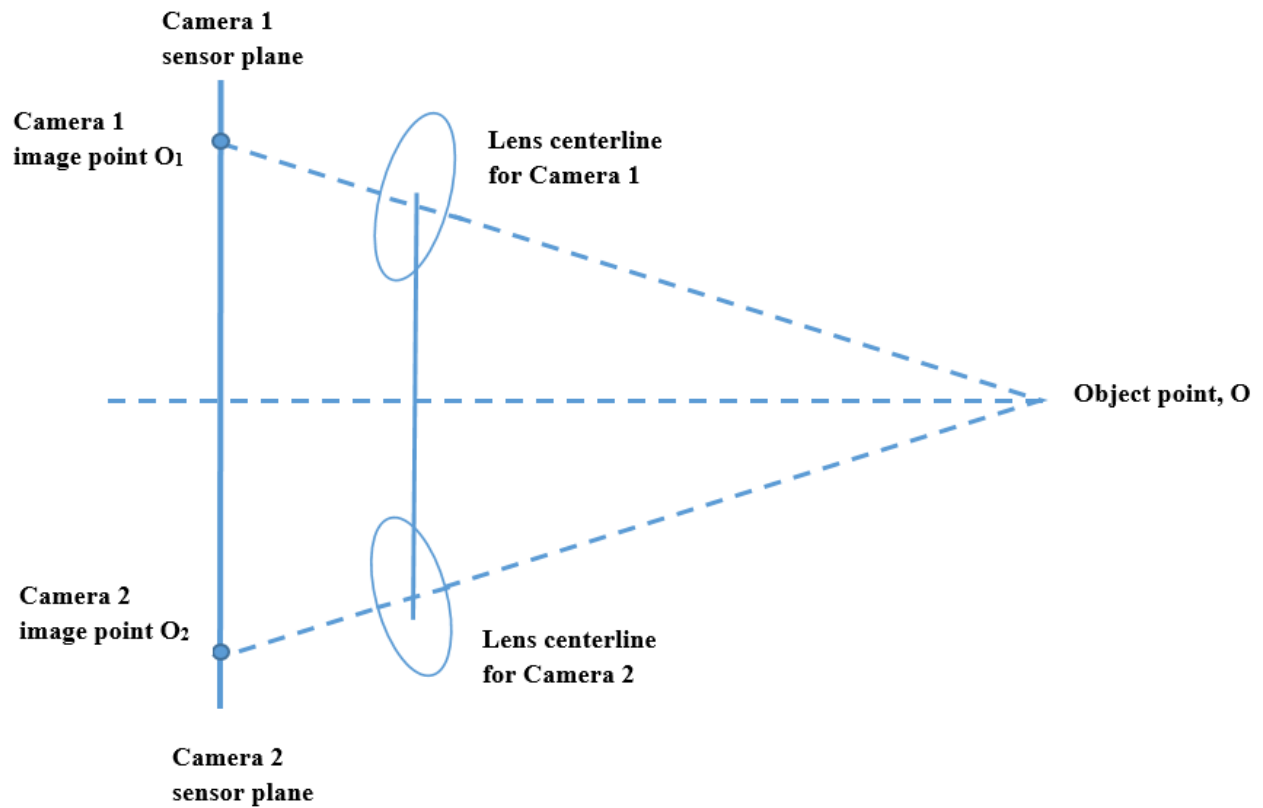

Figure 1: Top view schematic of a two-camera stereo vision system 


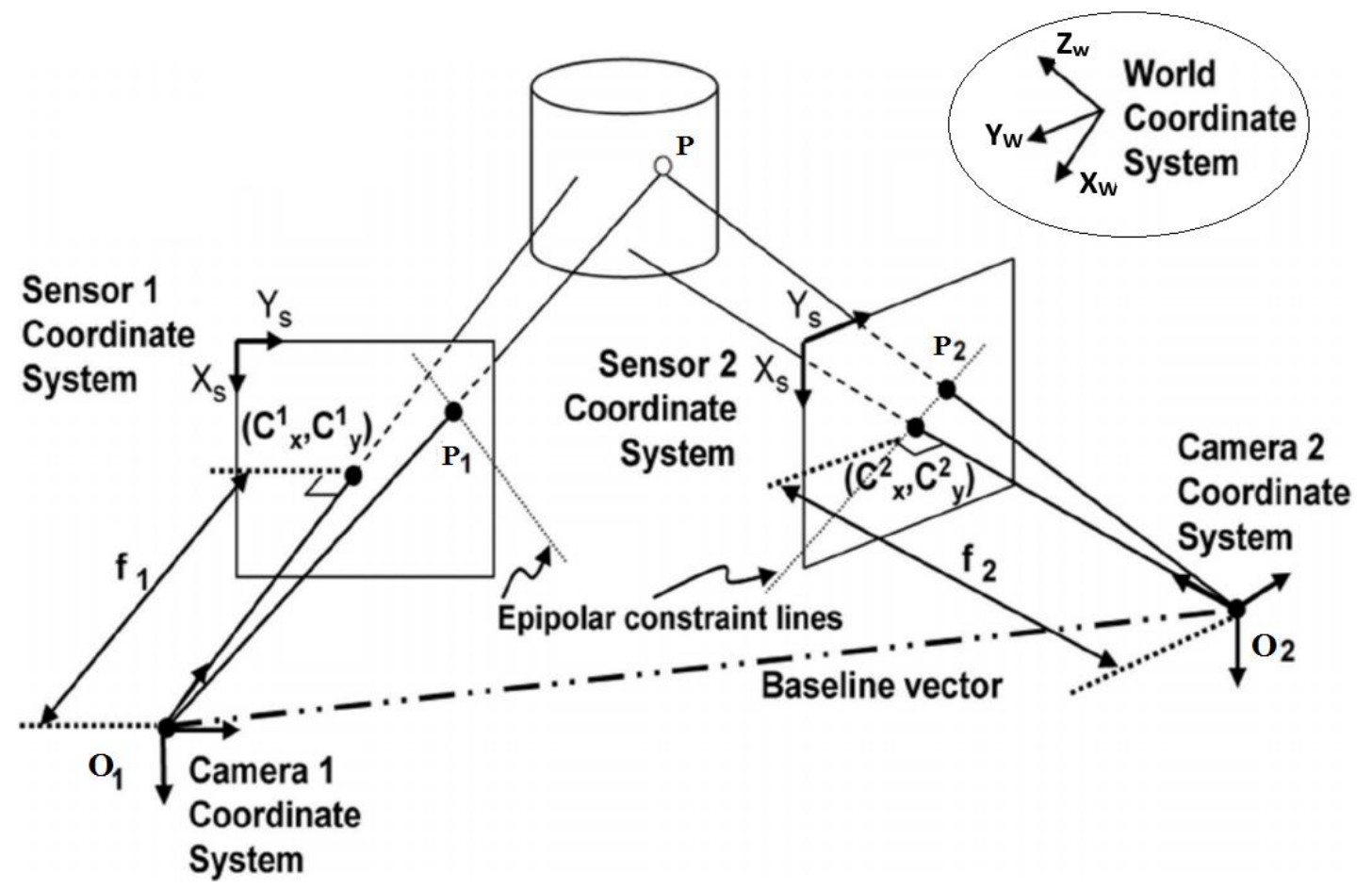

Figure 2 Schematic of the process used with a perspective projection model to convert stereo digital images into 3D measurements of position and displacement

Figure 2 shows a general schematic of a pair of standard pinhole cameras that are being used to map sensor locations in each camera to a 3D point $\mathrm{P}$ on an object. Figure 2 includes (a) a global and world coordinate system in the top right, (b) two sensor plane coordinate systems designated $\left(\mathrm{X}_{\mathrm{s}}, \mathrm{Y}_{\mathrm{s}}\right)$, (c) two optical axes with origins at the pinhole locations $\mathrm{O}_{1}$ and $\mathrm{O}_{2}$, (d) sensor plane center points $\left(\mathrm{C}_{\mathrm{x}}, \mathrm{C}_{\mathrm{y}}\right)$ for each camera corresponding to the intersection of the image plane with the optical axes, and (e) distance from origin of each camera system to the sensor plane, designated $f$. For a pinhole camera model, the "perspective projection" transformation for each camera shown graphically in Figure 2 can be used to relate 3D coordinates of a common point, $\mathrm{P}$, to sensor plane coordinates [15]:

$$
\begin{aligned}
X_{s} & =C_{1 x}+f_{1 x} \frac{R_{11} X_{W}+R_{12} Y_{W}+R_{13} Z_{W}+t_{1 x}}{R_{31} X_{W}+R_{32} Y_{W}+R_{33} Z_{W}+t_{1 z}}+s * \frac{R_{11} X_{W}+R_{12} Y_{W}+R_{13} Z_{W}+t_{1 x}}{R_{31} X_{W}+R_{32} Y_{W}+R_{33} Z_{W}+t_{1 z}} \\
Y_{s} & =C_{1 y}+f_{1 y} \frac{R_{21} X_{W}+R_{22} Y_{W}+R_{23} Z_{W}+t_{1 y}}{R_{31} X_{W}+R_{32} Y_{W}+R_{33} Z_{W}+t_{1 z}}
\end{aligned}
$$

For each camera, the parameters $f, C_{x}$ and $\mathrm{C}_{y}$ shown in Figure 2 are a portion of the set of intrinsic camera parameters that are solely a function of the camera-lens combination used in an experiment. Not shown directly in Figure 2 are three additional intrinsic camera parameters; image plane skew, $s$, and scale factors. The skew parameter, $s$, accounts for non- 
orthogonality of the sensor coordinates $\left(X_{s}, Y_{s}\right)$ and the scale factors $S_{x}$ and $S_{y}$, have units of pixels/metric length in the sensor plane (inverse of the physical size of the camera sensor) in both $X_{s}$ and $Y_{s}$ directions. The scale factors for each view do not appear separately but in multiplicative form with $f$ as $f_{x}=f S_{x}$ and $f_{y}=f S_{y}$, so that there are five independent intrinsic parameters $C_{x}, C_{y}, s, f_{x}$ and $f_{y}$. In addition to the five intrinsic parameters, there are a set of six extrinsic parameters relating the global coordinate system defining each object point, $\mathrm{P}$, to the pinhole systems located at $\mathrm{O}_{1}$ and $\mathrm{O}_{2}$, respectively. The extrinsic parameters include the rotation matrixs for each system, $[R]$, with three independent angles orienting the global system relative to each camera system and the translation vectors, $\mathbf{t}$, with three independent parameters relating the center of each camera to the global system. Thus, for an "ideal" pair of cameras there are 22 parameters to be determined so that one can relate the sensor coordinates $\left(X_{s}, Y_{s}\right)$ in both cameras to a $3 \mathrm{D}$ position in space.

To obtain all 22 camera parameters for the analytical analysis presented in Section 6, a bundle adjustment calibration process is employed using commercial software, VIC-3D [16]. In general, the calibration process is described as follows. Relative to a general world coordinate system, a W x N two-dimensional array of $3 \mathrm{D}$ points $\left(X_{W}, Y_{W}, Z_{W}\right)$ with a known spacing between individual points on a given target can be written:

$$
\vec{P}_{T}=\left(P_{1}, P_{2}, \ldots \ldots, P_{N}\right)=\left[\left(X_{w 1}, Y_{w 1}, Z_{w 1}\right) ;\left(X_{w 2}, Y_{w 2}, Z_{w 2}\right) ; \ldots \ldots .,\left(X_{w N}, Y_{w N}, Z_{w N}\right) .\right.
$$

When imaging any of the target points, say $P_{1}$, a metric measure of the difference between the model prediction of the projected sensor location using the pinhole camera model and the measured sensor location can be written:

$$
E\left(\operatorname{Int}, \operatorname{Ext}, p_{1}\right)=\left\|\left[x_{s}-X_{s}\left(\operatorname{Int}, \operatorname{Ext}, p_{1}\right)\right]^{2}+\left[y_{s}-Y_{s}\left(\operatorname{Int}, \operatorname{Ext}, p_{1}\right)\right]^{2}\right\|
$$

where the measured sensor location are denoted $\left(x_{s}, y_{s}\right)$ and the model-projected sensor location are denoted $\left(X, Y_{s}\right)$. Figure 3 shows a typical calibration target. If the target has $\mathrm{N}$ grid points and the target is moved to give $\mathrm{M}$ views of an object, then Equ (3) can be written in the following form [17]

$$
E\left(\operatorname{Int}, \operatorname{Ext}, p_{1}\right)=\sum_{J=1}^{M} \sum_{I=1}^{N}\left\|\left[x_{s}-X_{s}\left(\operatorname{Int}, \operatorname{Ext}, p_{T}\right)\right]^{2}+\left[y_{s}-Y_{s}\left(\operatorname{Int}, \operatorname{Ext}, p_{T}\right)\right]^{2}\right\|^{2}
$$

where $X_{s}\left(\right.$ Int $\left., E x t, p_{1}\right)$ is the model prediction of the measured $x_{s}$ location on the sensor plane for point $\mathrm{p}_{1}$; $Y_{s}\left(\right.$ Int, Ext, $\left.p_{1}\right)$ is the model prediction of the measured $y_{s}$ location on the sensor plane for point $\mathrm{p}_{1}$; $\mathrm{p}_{\mathrm{T}}$ are the $3 \mathrm{D}$ world coordinates $\left(X_{W}, Y_{W}, Z_{W}\right)$ 
of all $\mathrm{N}$ target points in all $\mathrm{M}$ views; Int includes the five intrinsic camera parameters for each camera; Ext includes not only the extrinsic parameters for each camera but also the six rotation and translation parameters for each position and orientation of the calibration target, changing for all $\mathrm{M}$ views of the calibration target.

Once the calibration images have been obtained and $\mathrm{N}$ target points are identified in all $\mathrm{M}$ views, the optimal values for all parameters are determined simultaneously by minimizing the error metric in Equ (4). Since global minimization across all parameters is performed, if the minimization process is not sensitive to a parameter then it is possible for some of the parameters to be assigned non-physical values. As an example, for high magnification stereo-vision studies, the optimization process may result in locations for the optimal lens center parameters that are far from the centerline of the sensor plane, simply due to the insensitivity of the minimum to these values. Conversely, for those parameters that have a significant impact on accurate identification of a three-dimensional position (e.g. focal lengths), any "bias" in the optimal values has the potential to cause increased errors in the predicted three-dimensional position of an object point.

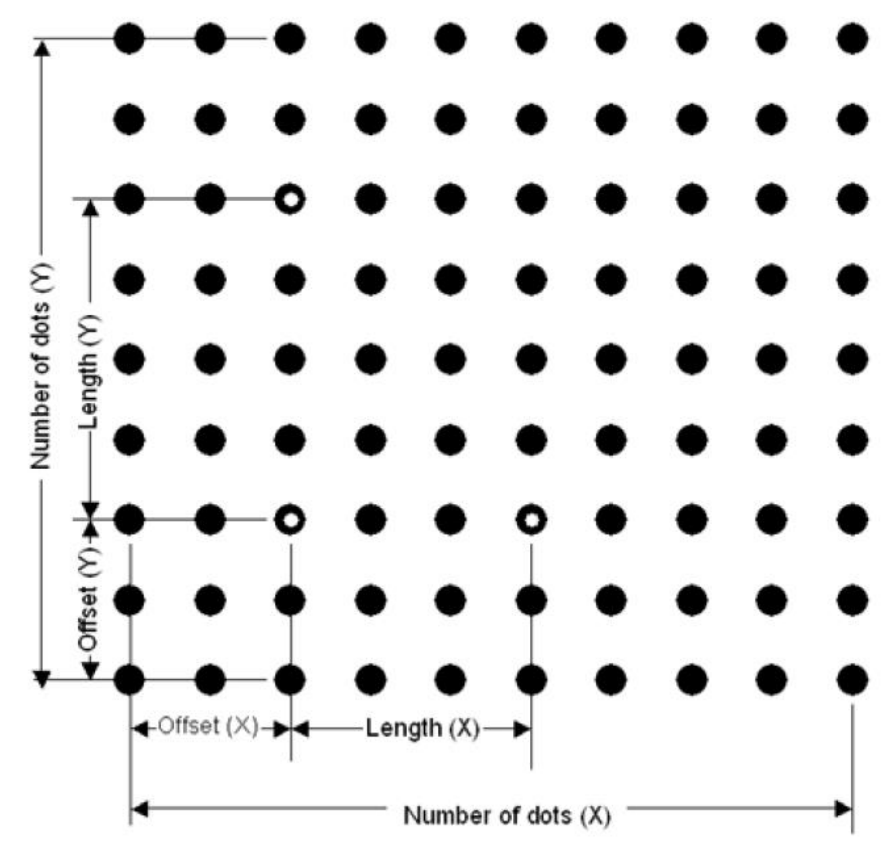

Figure 3 A typical calibration pattern used in our calibration process

\section{Sensitivity Analysis Theory}

As noted above, identification of those parameters that are most important for accurate three-dimensional measurements is important in assessing the source of inaccuracies in the stereo-vision measurements. Known as sensitivity analysis, the approach is used to explore calibration parameters that are most significant in the measurement of deformations using stereo-vision with digital image correlation. There are three different methods commonly used for sensitivity analysis; mathematical, statistical and graphical [18]. Mathematical methods determine sensitivity of a model output to the possible 
range of input parameters [19]. Statistical methods provides probability distributions of output parameter using variance in the input parameters [20]. Depending on the method of statistical distribution, one or more input parameters are varied at a time. Graphical methods depicts sensitivity in the form of graphs, charts, or surfaces. Generally, graphical methods are a useful tool to present the output for different input combinations [21]. In this study, Statistical method is used to investigate the effect of camera parameters on StereoDIC strain measurements when a planar specimen is subjected to rigid body out of plane rotation, $\theta$, in the range $-40^{\circ} \leq \theta \leq 40^{\circ}$. A parameter having high sensitivity indicates that slight changes in the camera parameter will have a significant effect on the deformation measurements (e.g. on the measurements for the strain $\varepsilon_{x x}$ ).

Variance based sensitivity analysis (often referred to as the Sobol method or Sobol indices, after Ilya M. Sobol [22]) is a form of global sensitivity analysis. Variance based measures of sensitivity are attractive because they measure sensitivity across the whole input space. As such, these measures can deal with nonlinear response as well as quantify the effect of interactions in non-additive systems [23].To recalls the basic concepts of variance based measures, consider a model outputs (Y) that a function of input parameters $\left(X_{1}, X_{2} \ldots \ldots X_{K}\right)$. This relationship can be written as follows:

$$
Y=f\left(X_{1}, X_{2} \ldots \ldots X_{K}\right)
$$

$Y$ is a chosen univariate model output. Furthermore, it will be assumed that the inputs are independently and uniformly distributed within the unit hypercube, i.e. $X_{i} \in[0,1]$ for $i=1,2, \ldots . ., k$. This incurs no loss of generality because any input space can be transformed onto this unit hypercube. $f(X)$ may be decomposed in the following way

$$
f=f_{0}+\sum_{i} f_{i}+\sum_{i} \sum_{j>1} f_{i j}+\ldots+f_{12 \ldots k}
$$

where $f_{0}$ is a constant and $f_{i}$ is a function of $X_{i}, f_{i j}$ a function of $X_{i}$ and $X_{j}$ etc. A condition of this decomposition is

$$
\int_{0}^{1} f_{i 1, i 2, \ldots \ldots i s}\left(X_{i_{1}}, X_{i_{2}}, \ldots, X_{i_{s}}\right) d X_{i_{w}}=0, \text { for } i_{w} \in\left\{i_{1}, i_{2}, \ldots \ldots, i_{s}\right\}
$$

i.e. all the terms in the functional decomposition are orthogonal. This leads to definitions of the terms of the functional decomposition in terms of conditional expected values:

$$
\begin{aligned}
& f_{0}=E(Y) \\
& f_{i}\left(X_{i}\right)=E\left(Y \mid X_{i}\right)-f_{0} \\
& f_{i j}\left(X_{i}, X_{j}\right)=E\left(Y \mid X_{i}, X_{j}\right)-f_{0}-f_{i}-f_{j}
\end{aligned}
$$


From which it can be seen that $f_{i}$ is the effect of varying $X_{i}$ alone and $f_{i j}$ is the effect of varying $X_{i}$ and $X_{j}$ simultaneously, additional to the effect of their individual variations. This is known as a second-order interaction model, which is used in our studies.

Further assuming that $f(X)$ is square integrable, the functional decomposition may be squared and integrated to give,

$$
\int_{0}^{1} f^{2}(X) d X-f_{0}^{2}=\sum_{s=1}^{k} \sum_{i_{1}<\ldots<i_{s}}^{k} f_{i_{1} \ldots i_{s}}^{2} d X_{i_{1}} \ldots d X_{i_{s}}
$$

Notice that the left hand side is equal to the variance of $Y$, and the terms of the right hand side are variance terms, now decomposed with respect to sets of the $X_{i}$. This finally leads to the decomposition of variance expression,

$$
V(Y)=\sum_{i} V_{i}+\sum_{i} \sum_{j>1} V_{i j}+\ldots .+V_{12 \ldots . k}
$$

where,

$$
\begin{aligned}
V_{i} & =\operatorname{Var}_{X i}\left[E_{X \sim i}\left(Y \mid X_{i}\right)\right], \\
V_{i j} & =\operatorname{Var}_{X_{i j}}\left[E_{X \sim i j}\left(Y \mid X_{i}, X_{j}\right)\right]-V_{i}-V_{j}
\end{aligned}
$$

and so on for higher order terms.

The $X_{\sim i}$ notation indicates the set of all variables except $X_{i}$. The above variance decomposition shows how the variance of the model output can be decomposed into terms attributable to each input, as well as the interaction effects between them. Together, all terms sum to the total variance of the model output.

Dividing both sides of the Equ(10) by V (Y), we obtain:

$$
\sum_{i} S_{i}+\sum_{i} \sum_{j>1} S_{i j}+\ldots . S_{12 \ldots . k}=1
$$

Where $\sum_{i} S_{i}=\frac{\sum_{i} V_{i}}{V(Y)}$ is the sum of first order indices

$\sum_{i} \sum_{j>1} S_{i j}=\frac{\sum_{i} \sum_{j>1} V_{i j}}{V(Y)}$ is the sum of second order indices and so on 
$\mathrm{Equ}(12)$ is valid when all input parameters are independent of each other. Due to the ratio of partial variances (e.g. Vi, $V i j$, etc) to total variance $(\mathrm{V}(\mathrm{Y}))$, all the sensitivity indices are scaled between 0 and 1 interval. When the summation of all first order indices gives unity, that is, $\sum_{i} S_{i}=1$, the model is known as additive, that is, without any interaction effect. Hence, $1-\sum_{i} S_{i}$ indicates interaction effects that could either be one or a combination of second order, or higher order. The total effect index, $\mathrm{S}_{\mathrm{Ti}}$, for each input parameter is given by:

$$
S_{T i}=S_{i}+\sum_{i \neq j} S_{i j}+\sum_{i \neq j \neq l} S_{i j l}+\ldots
$$

For instance, if $\mathrm{k}=3$ then the total effect index would be given by:

$S_{T 1}=S_{1}+S_{12}+S_{13}+S_{123}$

where $S_{1}$ is first order index of input parameter $1, S_{12}$ is second order index of interaction effect between input parameters 1 and 2, $S_{13}$ is second order index of parameters 1 and 3, and $S_{123}$ is third order index of interaction effect between input parameters 1,2 and 3. The same method is applied for decomposition of $S_{T 2}$ and $S_{T 3}$ total effect index. Since $S_{T i}$ includes first to higher order relating to input parameter i, $S_{T i}-S_{i}$ indicates the interaction effect that only accounts for second and higher order indices.

First order sensitivity index and total effect index were estimated by quasi- Monte Carlo estimators (Saltelli et al. 2010):

$$
\begin{aligned}
& S_{i}= \frac{\left[(1 / N) \sum_{m=1}^{N}\left(y_{A}^{(m)}\right)^{2}-f_{0}^{2}\right]-\left[(1 / 2 N) \sum_{m=1}^{N}\left(y_{B}^{(m)}-y_{C i}^{(m)}\right)^{2}\right]}{\left[(1 / N) \sum_{m=1}^{N}\left(y_{A}^{(m)}\right)^{2}-f_{0}^{2}\right]} \\
& S_{T i}=\frac{\left.(1 / 2 N) \sum_{m=1}^{N}\left(y_{A}^{(m)}-y_{C i}^{(m)}\right)^{2}\right]}{\left[(1 / N) \sum_{m=1}^{N}\left(y_{A}^{(m)}\right)^{2}-f_{0}^{2}\right]}
\end{aligned}
$$

Both $y_{A}^{(m)}$ and $y_{B}^{(m)}$ are model outputs, as shown in Eq. (14) and (15). Sobol' quasi-random sequences were used to generate two sets of data, that is, matrix A and B corresponding to model outputs of $y_{A}^{(m)}$ and $y_{B}^{(m)}$ and these dataset were confined 
between 0 and 1 . The $f_{0}^{2}$ is given by $\left((1 / N) \sum_{m=1}^{N} y_{A}^{(m)}\right)^{2}$. The $y_{C i}^{(m)}$ is also model output; all the dimensions in the matrix were taken from matrix A, except i column, the dimension which is taken from matrix B.

\section{Experimental Considerations}

To provide quantitative data regarding the accuracy of StereoDIC strain measurements when a specimen is subjected to rigid body out of plane rotation, a series of experiments is performed. Figure 4 shows the experimental setup. Two LED light sources illuminate the specimen at the angles shown to minimize the potential for reflections. Two cameras are mounted firmly to an optical bench to eliminate unwanted camera motions during the experiment. The distance from the camera lenses to the specimen is approximately $0.30 \mathrm{~m}$. Table 1 summarizes the components in the stereovision system.

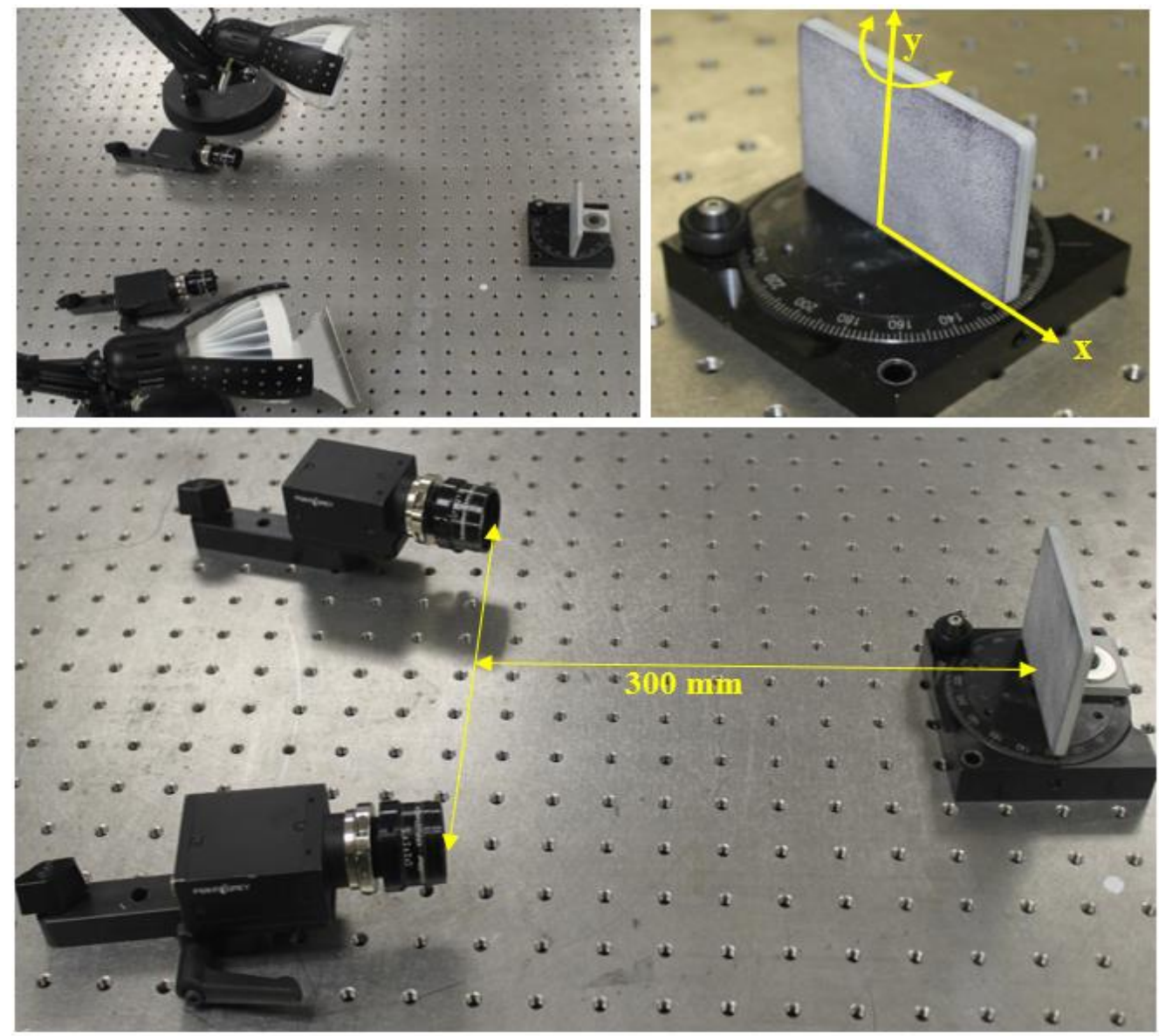

Figure 4 Experimental setup for out-of-plane rotation experiment. 
Table 1 Optical system components ${ }^{1}$

\begin{tabular}{|c|c|}
\hline & Stereovision system components \\
\hline Cameras & Grasshopper3 GS3-U3-91S6M (8bits, 3376X2704) \\
\hline Lenses & Schneider XENOPLAN 1.9/35-0511 \\
\hline Lighting & White LED Lighting \\
\hline Environment & Laboratory conditions, $\sim 20^{\circ} \mathrm{C}$ \\
\hline
\end{tabular}

To determine the effect of calibration procedures on the accuracy of stereovision system measurements in the presence of large rotations, two independent calibrations processes are performed. Designating the first calibration process as "Small Angle Calibration" (SAC), a total of 61 images of a translated and rotated planar calibration target with 12 x 9 dots on a $4 \mathrm{~mm}$ spacing (see Figure 3 for a typical 10X9 pattern) were acquired, where the rotation angles in all directions were less than $10^{\circ}$. Since the target spanned the majority of the field of view, translations were performed as needed so that calibration target images overlapped the entire field of view. Designating the second calibration process as "Large Angle Calibration" (LAC), a total of 142 images are used, where the first 61 images used for SAC are supplemented with 81 images of the same grid as it is rotated out-of-plane in $5^{\circ}$ increments from. $-40^{\circ} \leq \theta \leq 40^{\circ}$. Table 2 and Table 3 show the intrinsic and extrinsic calibration parameters, respectively, for both cameras that are obtained using the SAC and LAC processes described above.

Table 2 Calibration parameters for stereovision system

\begin{tabular}{|c|c|c|c|c|}
\hline Parameter & Cam1 - SAC & Cam2 - SAC & Cam1 - LAC & Cam2 - LAC \\
\hline$C_{x}$ (pixels) & $1676.743 \pm 67.1$ & $1712.878 \pm 82.913$ & $1656.108 \pm 22.86$ & $1728.664 \pm 14.72$ \\
\hline$C_{y}$ (pixels) & $1372.491 \pm 52.6$ & $1432.081 \pm 52.647$ & $1359.802 \pm 23.45$ & $1430.964 \pm 20.07$ \\
\hline$f \lambda_{x}$ (pixels) & $11176.322 \pm 66$. & $11134.508 \pm 64.68$ & $11140.057 \pm 4.42$ & $11114.128 \pm 4.55$ \\
\hline$f \lambda_{y}$ (pixels) & $11175.219 \pm 65$. & $11133.544 \pm 64.58$ & $11138.960 \pm 4.39$ & $11112.592 \pm 4.47$ \\
\hline$S$ (pixels) & $-3.742 \pm 0.279$ & $2.538 \pm 0.279$ & $-1.635 \pm 0.116$ & $0.958 \pm 0.116$ \\
\hline$\kappa 1$ (pixels ${ }^{-2}$ ) & $-0.273 \pm 0.000$ & $-0.225 \pm 0.000$ & $-0.271 \pm 0.000$ & $-0.223 \pm 0.000$ \\
\hline$\kappa 2\left(\right.$ pixels $^{-4}$ ) & 0 & 0 & 0 & 0 \\
\hline$\kappa 3$ pixels $^{-6}$ ) & 0 & 0 & 0 & 0 \\
\hline
\end{tabular}

\footnotetext{
${ }^{1}$ The experiments described in this section are repeated six times, with similar results obtained in all six experiments. In each case, the cameras were completely removed from the setup, reassembled and refocused on the specimen to ensure that the trends observed in the data could be observed in each case. The results for a typical experiment are reported in this work.
} 
Table 3 Camera $1 \rightarrow 2$ transformation parameters for stereovision system

\begin{tabular}{|c|c|c|}
\hline Camera $1 \rightarrow 2$ transformation & SAC & LAC \\
\hline$\alpha\left(^{\circ}\right)$ & $0.288^{\circ} \pm 0.0000$ & $0.341^{\circ} \pm 0.000$ \\
\hline$\beta\left(^{\circ}\right)$ & $15.966^{\circ} \pm 0.000$ & $15.815^{\circ} \pm 0.000$ \\
\hline$\gamma\left(^{\circ}\right)$ & $1.489^{\circ} \pm 0.000$ & $1.482^{\circ} \pm 0.000$ \\
\hline $\mathrm{T}_{\mathrm{x}}(\mathrm{mm})$ & $-77.473 \pm 0.002$ & $-77.412 \pm 0.000$ \\
\hline $\left.\mathrm{T}_{\mathrm{y}} \mathrm{mm}\right)$ & $-0.992 \pm 0.000$ & $-1.006 \pm 0.000$ \\
\hline $\mathrm{T}_{\mathrm{z}}(\mathrm{mm})$ & $10.229 \pm 0.025$ & $10.487 \pm 0.003$ \\
\hline Baseline $(\mathrm{mm})$ & $78.1521 \pm 0.025$ & $78.1256 \pm 0.003$ \\
\hline
\end{tabular}

Inspection of the camera parameters in Table 2 and Table 3 shows that the intrinsic calibration parameters including $C_{x}, C_{y}, f \lambda_{x}, f \lambda_{y}$ have larger variability for the $\mathrm{SAC}$ than $\mathrm{LAC}$, whereas some other parameters $(\alpha, \beta, \gamma)$ have variability less that $10^{-3}$. It should be noted that a first-order radial distortion model is used during calibration for both camera models. In the entire central $1 / 3$ region of the region, the radial corrections are less than 0.01 pixels (strains less than $25 \mu \varepsilon$ ). As will be shown, this level is much smaller than the effect being observed. Thus, a model that does not include radial distortion for the central region will not appreciably alter the measurement trends.

Once calibration is completed, the planar aluminum specimen is lightly coated with flat white paint and then speckled using a can of flat black spray paint. The resulting speckle pattern and grey level histogram for the recorded pattern are shown in Figure 5. The as-patterned specimen is then attached to the rotation stage shown in Figure 4. Care is taken to align the front surface of the specimen with the center of rotation so that each experiment approximates rigid body out of plane rotation. Once mounted in the rotation fixture, a total 15 images are acquired without rotation and after each $5^{\circ}$ rotation is completed within the range $-40^{\circ} \leq \theta \leq 40^{\circ}$. Strains are determined along the line shown in Figure 6 using experimental $U, V, W$ displacements and the following equation:

$$
\varepsilon_{x x}=\frac{\partial U}{\partial X}+\frac{1}{2}\left[\left(\frac{\partial U}{\partial X}\right)^{2}+\left(\frac{\partial V}{\partial X}\right)^{2}+\left(\frac{\partial W}{\partial X}\right)^{2}\right]
$$

Table 4 shows the 3D DIC parameters used to analyze the images. 

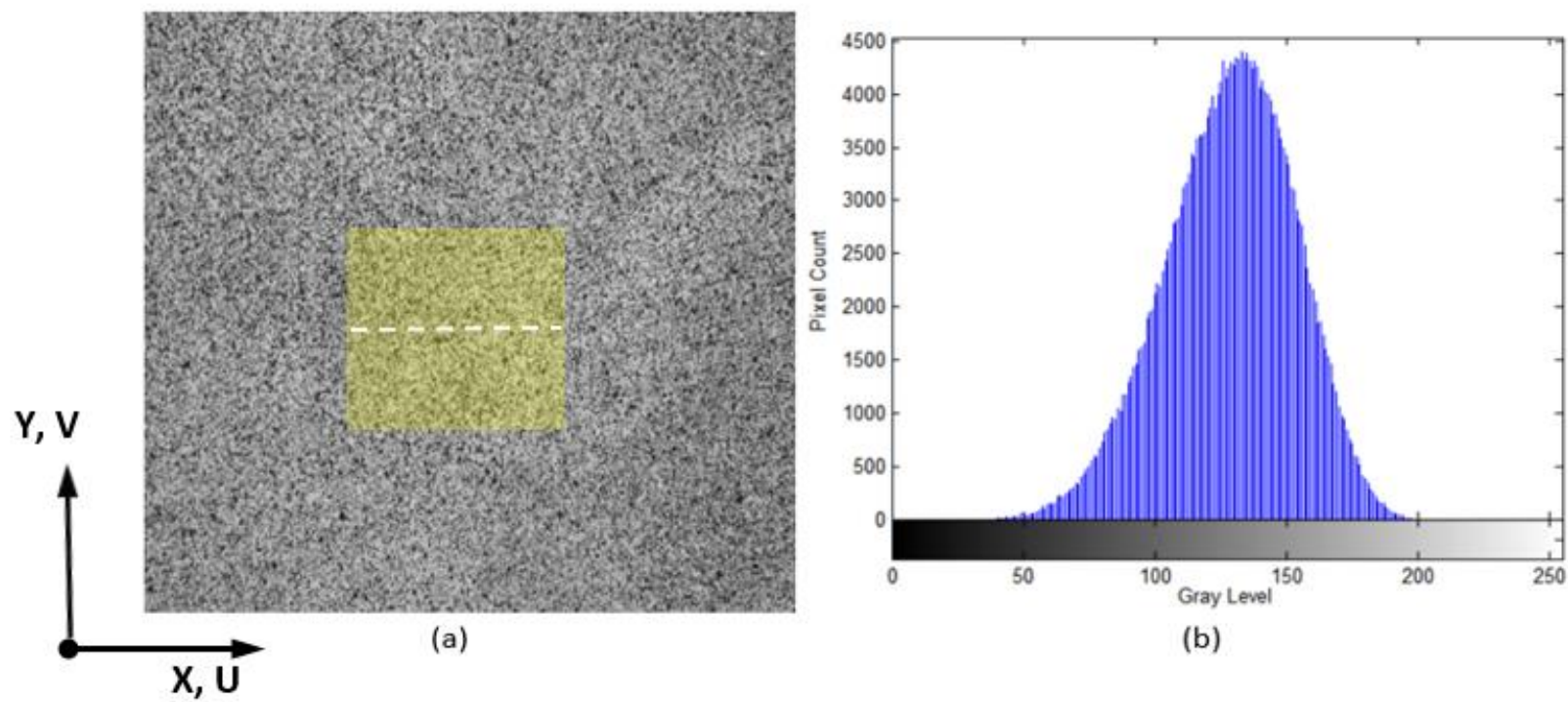

(b)

Figure 5 Speckle Pattern (a) image with central 900x800 pixel2 region where data is analyzed and (b) grey level histogram for pattern. Region of interest is shown in light yellow. Dashed line within yellow region indicates where points are acquired for theoretical predictions of strain.

Table 4 Parameters used for analysis of images

\begin{tabular}{|c|c|}
\hline \multicolumn{2}{|c|}{ StereoDIC Analysis Parameters } \\
\hline Subset size & 47 X 47 pixels $^{2}$ \\
\hline Step size & 13 pixels \\
\hline Shape function & Affine \\
\hline Software & VIC-3D \\
\hline Strain Filter Size & $5 \times 5$ \\
\hline
\end{tabular}

To estimate variability in the measurements using StereoDIC, the central 1/3 region of the fifteen images of the specimen acquired without out-of-plane rotation is analyzed (see Fig. 5). The standard deviations in the displacements with no motion using LAC and SAC parameters are shown in Table 5. Inspection of Table 5 shows that the $\mathrm{W}$-displacement under no rotation has slightly lower variability for the SAC calibration, even though the range for out-of-plane rotations during calibration is smaller. 
Table 5 Variability in displacement components at no rotation condition

\begin{tabular}{|c|c|c|}
\hline Displacement component & Standard deviation (LAC) & Standard deviation (SAC) \\
\hline $\mathrm{U}$ & $\pm 0.0007288 \mathrm{~mm}$ & $\pm 0.000722 \mathrm{~mm}$ \\
\hline $\mathrm{V}$ & $\pm 0.000539 \mathrm{~mm}$ & $\pm 0.000523 \mathrm{~mm}$ \\
\hline $\mathrm{W}$ & $\pm 0.004092 \mathrm{~mm}$ & $\pm 0.003706 \mathrm{~mm}$ \\
\hline
\end{tabular}

\section{Experimental Results and Discussion}

Figures 6 and 7 show contour plots in the central 1/3 portion of the specimen for displacement components (U, V, W) for out of plane rotations, $\phi=-40^{\circ},-20^{\circ}, 0^{\circ}, 20^{\circ}$ and $40^{\circ}$ using LAC and SAC parameters, respectively. Figure 8 and 9 show the displacements $(\mathrm{U}, \mathrm{V}, \mathrm{W})$ along the line $\mathrm{L}_{\mathrm{o}}$ when using LAC and SAC parameters for $40^{\circ}$ out-of-plane clockwise and anticlockwise rotations, respectively. Figure 10 shows the average in-plane strain $\varepsilon_{x x}$ along line $\mathrm{L}_{0}$ as a function of out-ofplane rotation angle using SAC and LAC process when the specimen undergoes rigid body out-of-plane rotation.

As shown in the contour plots in Figures 6 and 7, all three displacement components have gradients primarily in the X-direction (horizontal); this is the direction along which points move towards and away from the camera due to rotation of the specimen. Figure 8 and Figure 9 show that the gradients in all displacement components are linear along $\mathrm{L}_{\mathrm{o}}$. Figure 10 shows that the average strain measurements using SAC calibration result in a parabolically increasing strain $\varepsilon_{\mathrm{xx}}$, reaching a maximum of $\approx 1400 \mu \varepsilon$ for the largest rotation angles. For LAC calibration, there is a weak parabolic increase in strain, $\varepsilon_{\mathrm{xx}}$, reaching a maximum of $\approx 300 \mu \varepsilon$ for the largest rotation angles. Inspection of the linear trends in Figures 8 and 9 and Equ (16) demonstrates that both $\partial \mathrm{U} / \partial \mathrm{X}$ and $\partial \mathrm{W} / \partial \mathrm{X}$ contribute nearly equally to the strain calculation, suggesting that the measured strain errors are due to inaccuracies in a combination of $\mathrm{U}$ and $\mathrm{W}$ displacements along the $\mathrm{X}$-direction.

Regarding the reason for the large measurement errors for the SAC calibration process, several experimental reasons for the errors can be conjectured including specimen defocus, loss of speckle pattern contrast in at least one of the camera images and changes in lighting due to rotation. In this work, images are analyzed and (a) found to be in focus, (b) the lighting is shown to be relatively uniform in central region for all angles and (c) contrast in the images for all rotation angles of rotation is measured and shown to be adequate. Beyond all of the potential error sources that are excluded in our studies, another distinct possibility is that there is bias in some/all of the calibration parameters for SAC calibration. To determine whether the trends shown in Figure 10 are consistent with theoretical predictions, a set of simulations are performed using Equ (2) to determine whether the effect can be attributed to the difference in camera parameters obtained by the SAC and LAC processes. 
(a)
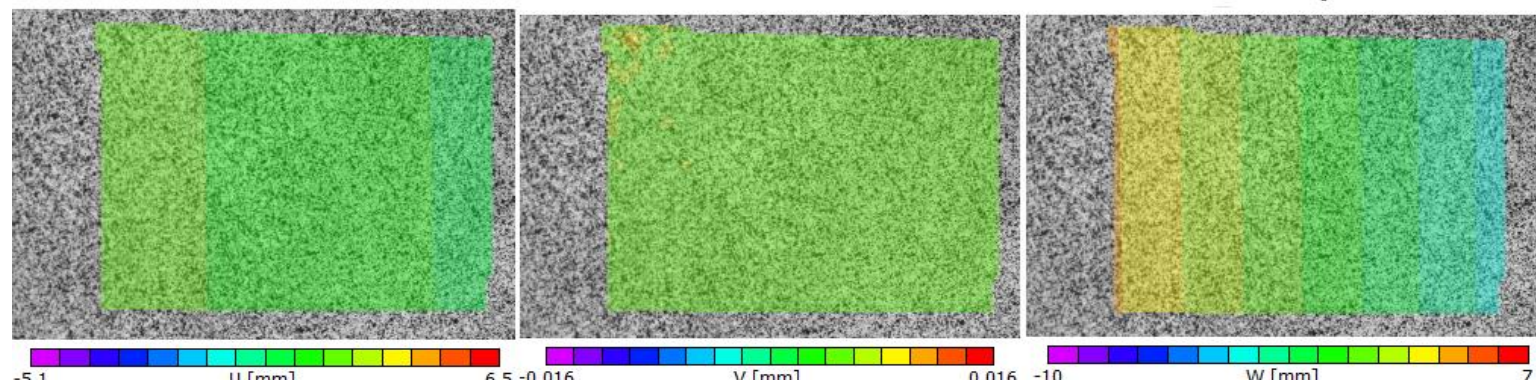

$-5.1$

$U[\mathrm{~mm}]$

$6.5-0.016$

$v[\mathrm{~mm}]$

$\frac{\mid}{W[\mathrm{~mm}]}$
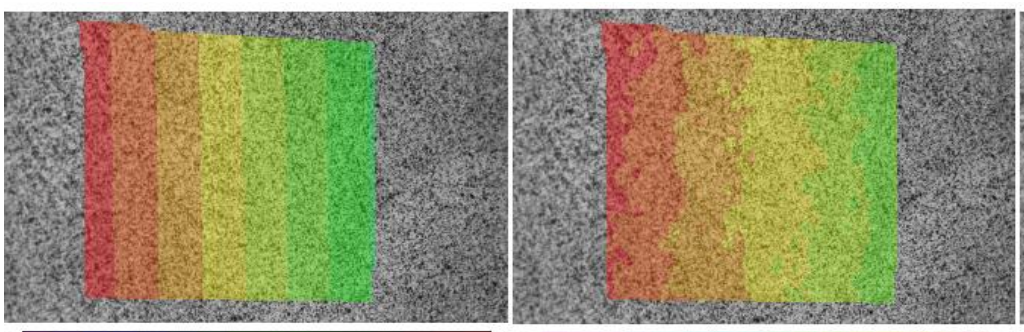

$-5.1$

$U[\mathrm{~mm}]$

$6.5-0.016$

$\frac{\mid}{v[\mathrm{~mm}]}$

$0.016-10$
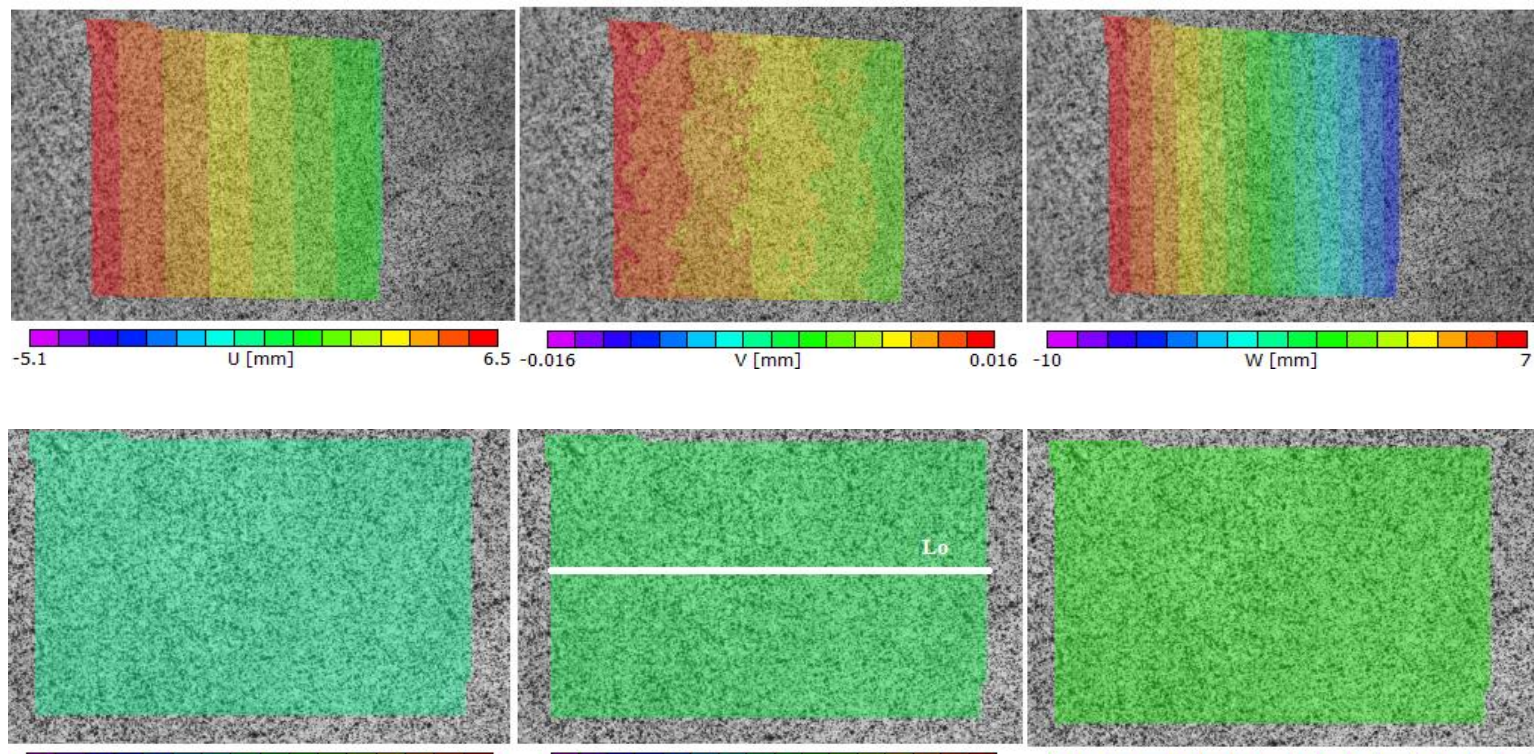

$6.5-0.016$

$v[\mathrm{~mm}]$

$0.016-10$

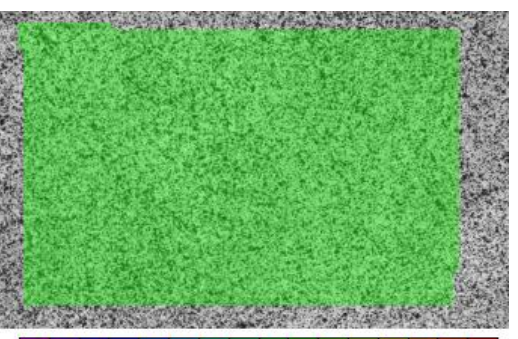

$-5.1$

$\cup[\mathrm{mm}]$
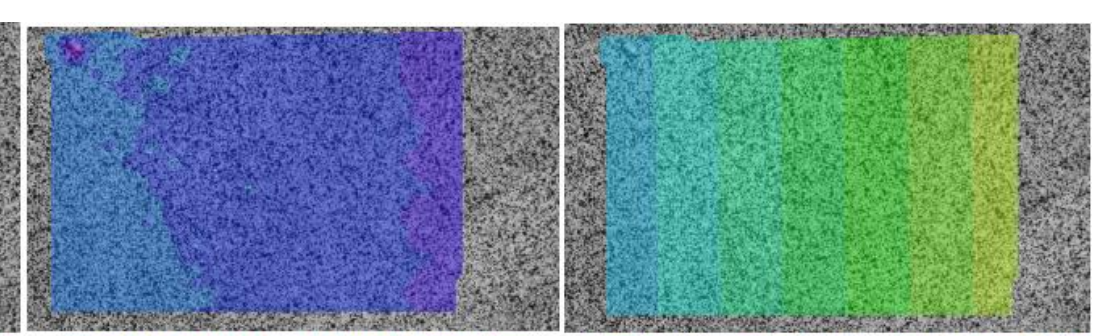

$-5.1$

$\begin{array}{ll}6.5 & -0.016\end{array}$

$\mathrm{V}[\mathrm{mm}]$

$0.016-10$

$\mathrm{W}[\mathrm{mm}]$

(e)
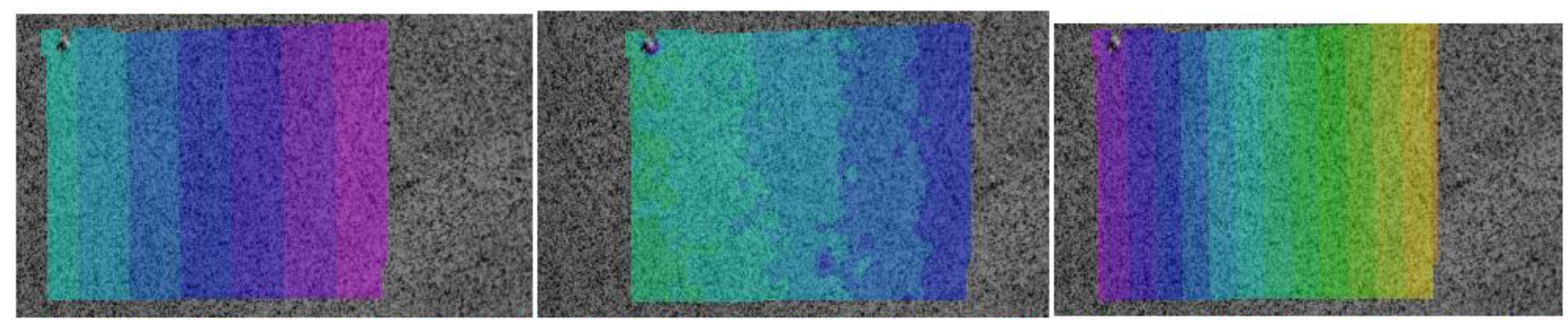

$-5.1$

$U[\mathrm{~mm}]$

$6.5-0.016$

$\mathrm{V}[\mathrm{mm}]$

$0.016-10$

$W[\mathrm{~mm}]$

Figure 6 Typical $U, V, W$ displacement contours obtained using VIC-3D for large angle calibrated (LAC) stereo pair for out-of-plane rotation. (a) $\theta=20^{\circ}$; (b) $\theta=40^{\circ}$; (c) $\theta=0^{\circ}$; (d) $\theta=-20^{\circ}$;(e) $\theta=-40^{\circ}$; All units in mm. 
(a)

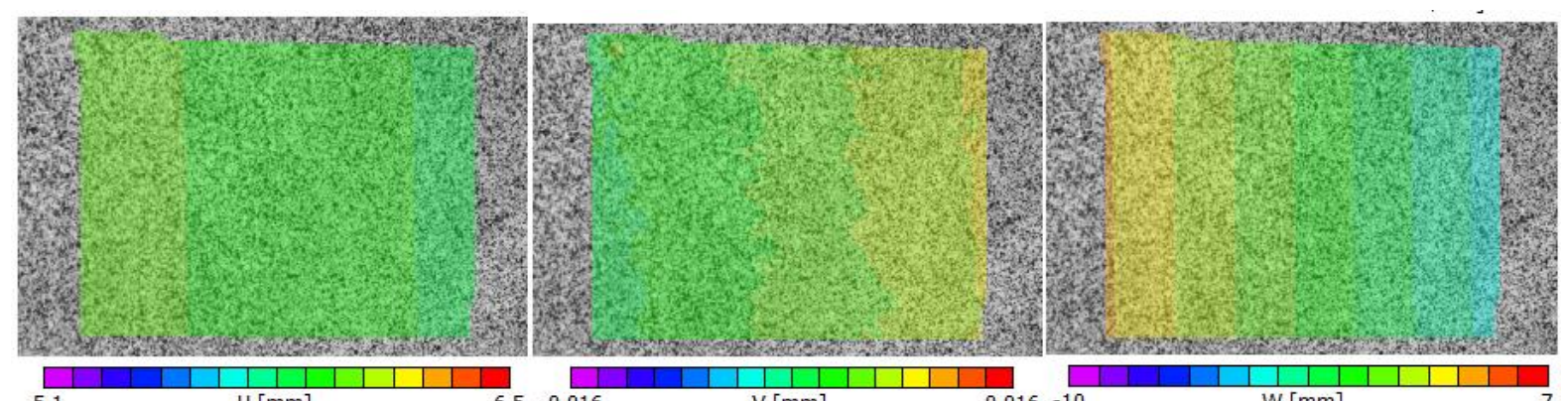

$-5.1$

$\mathrm{U}[\mathrm{mm}]$

$6.5-0.016$

$V[\mathrm{~mm}]$

$0.016-10$

$\mathrm{W}[\mathrm{mm}]$
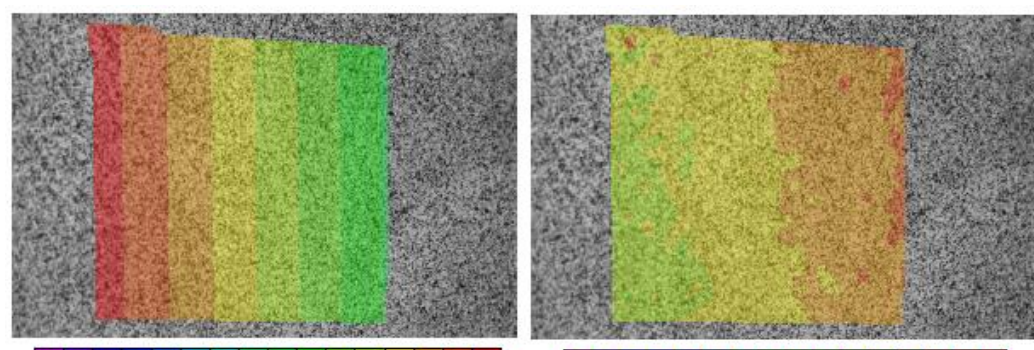

$-5.1$

$U[\mathrm{~mm}]$

$6.5-0.016$

$\mathrm{V}[\mathrm{mm}]$

$0.016-10$
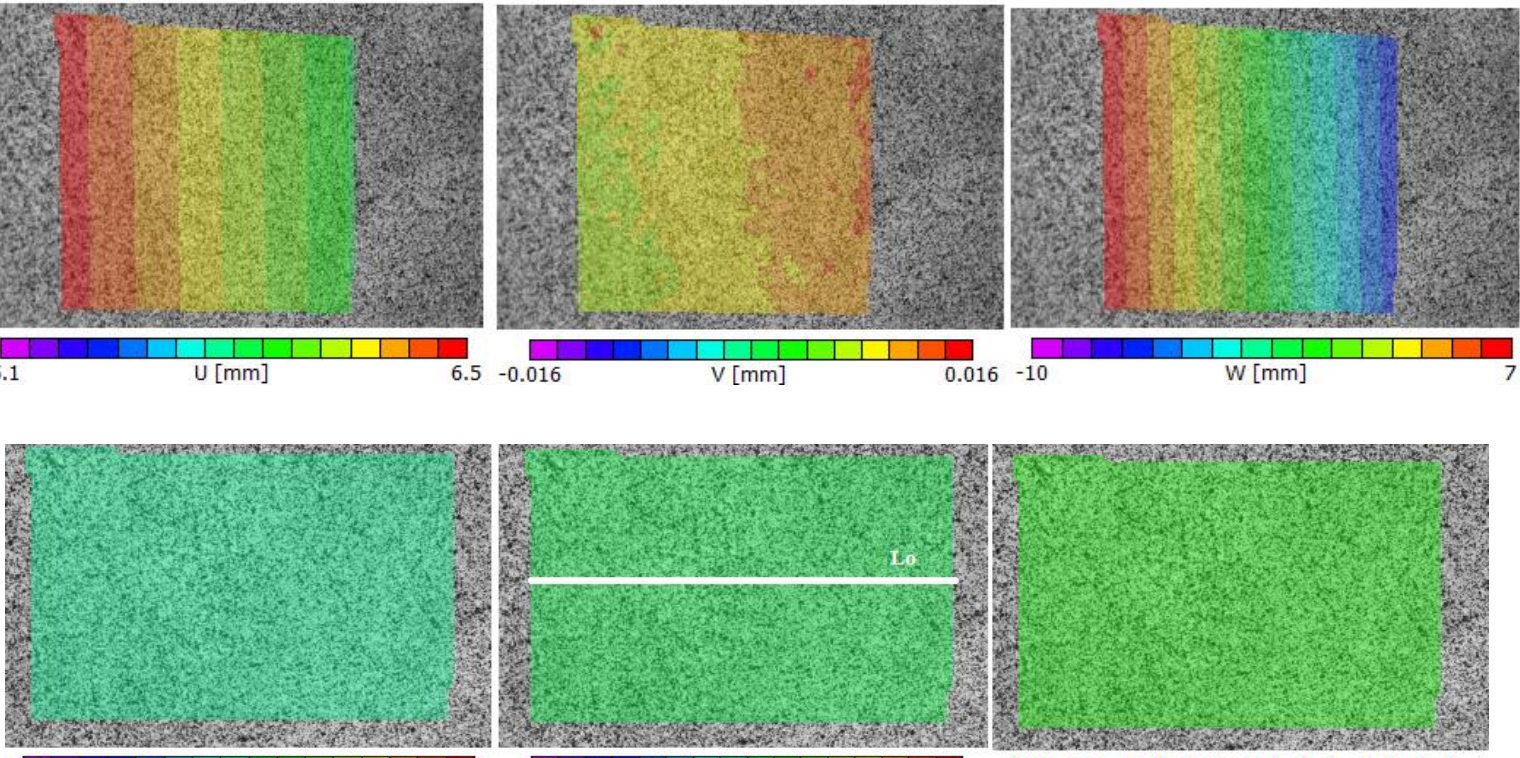

$-5.1$

$\cup[\mathrm{mm}]$

$\begin{array}{lr}-5 & -0.016\end{array}$

$\frac{\mid}{v[\mathrm{~mm}]}$

$\mathbb{0 . 0 1 6}_{-1}$

$\frac{|\mathrm{I}|}{\mathrm{W}[\mathrm{mm}]}$
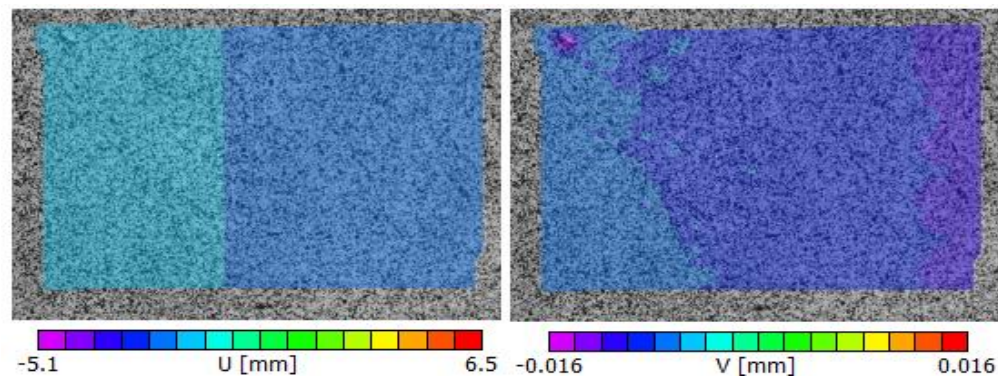

$\begin{array}{ll}6.5 & -0.016\end{array}$

$v[\mathrm{~mm}]$

$0.016-10$

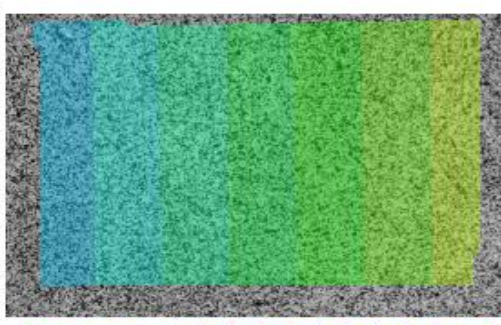

10

$\mathrm{W}[\mathrm{mm}]$
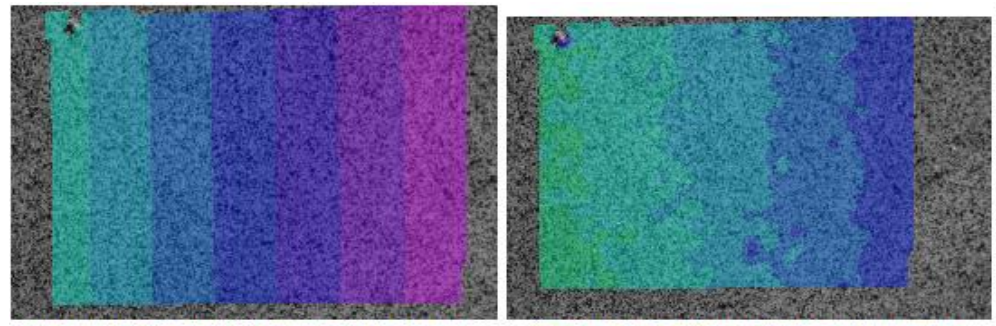

$-5.1$

$\mathrm{U}[\mathrm{mm}]$

$6.5-0.016$

$\mathrm{V}[\mathrm{mm}]$

$\square_{0.016}$

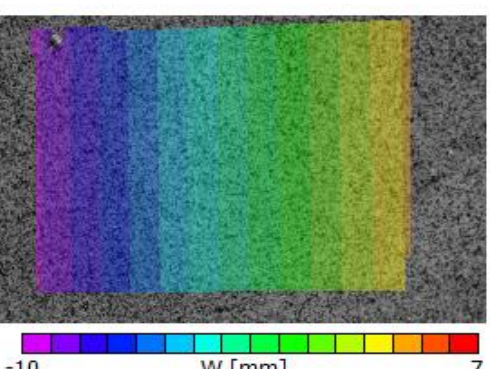

Figure 7 Typical $U, V, W$ displacement contours obtained using VIC-3D for small angle calibrated (SAC) stereo pair for out-

of-plane rotation (a) $\theta=20^{\circ}$, (b) $\theta=40^{\circ}$, (c) $\theta=0^{\circ}$; (d) $\theta=-20^{\circ}$; (e) $\theta=-40^{\circ}$. All units in mm. 

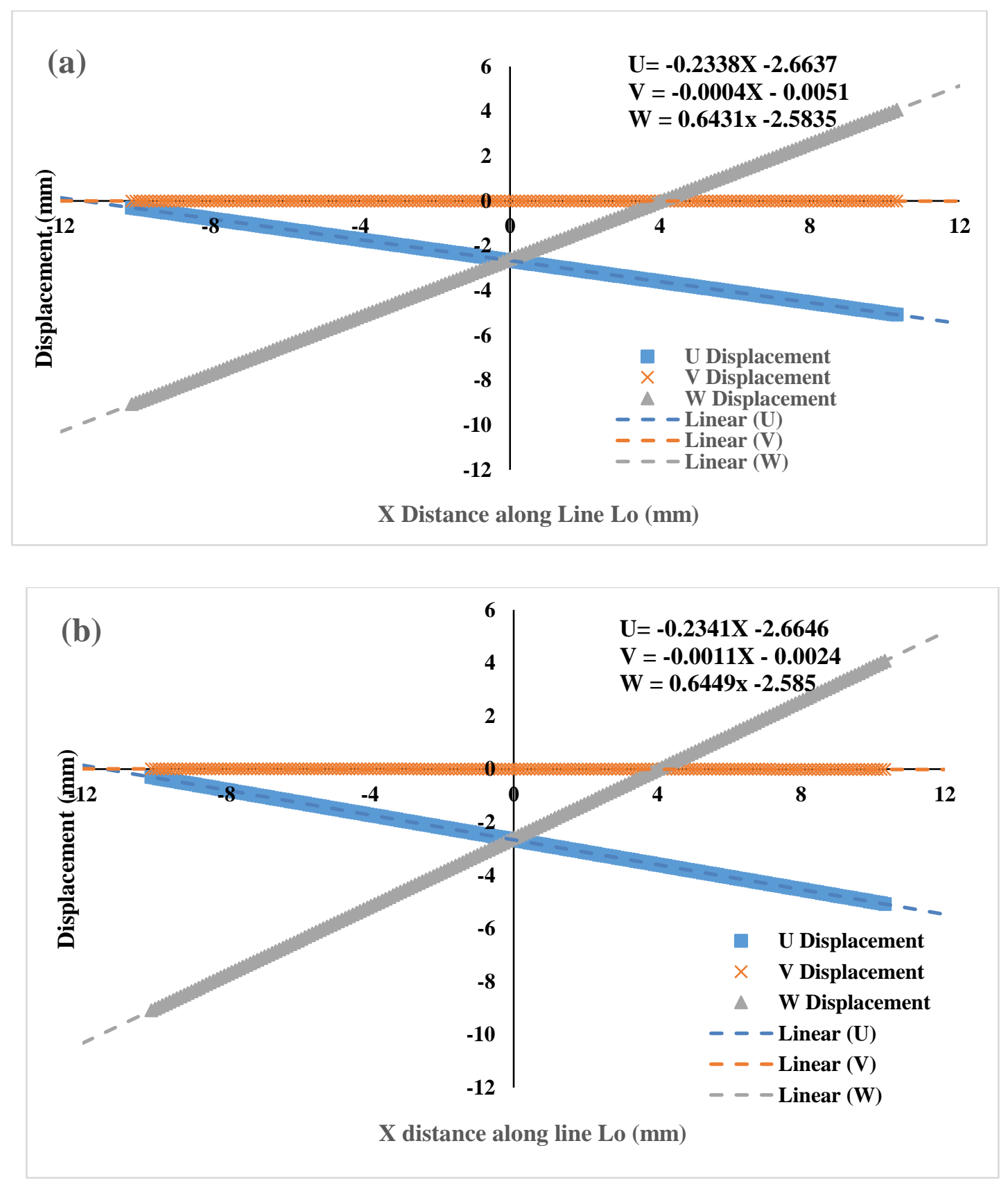

Figure 8 Typical $U, V, W$ displacement fields obtained using VIC-3D along line $L_{0}$ for (a) LAC (b) SAC stereo pair for out-of-plane rotation $\theta=-40^{\circ}$. 

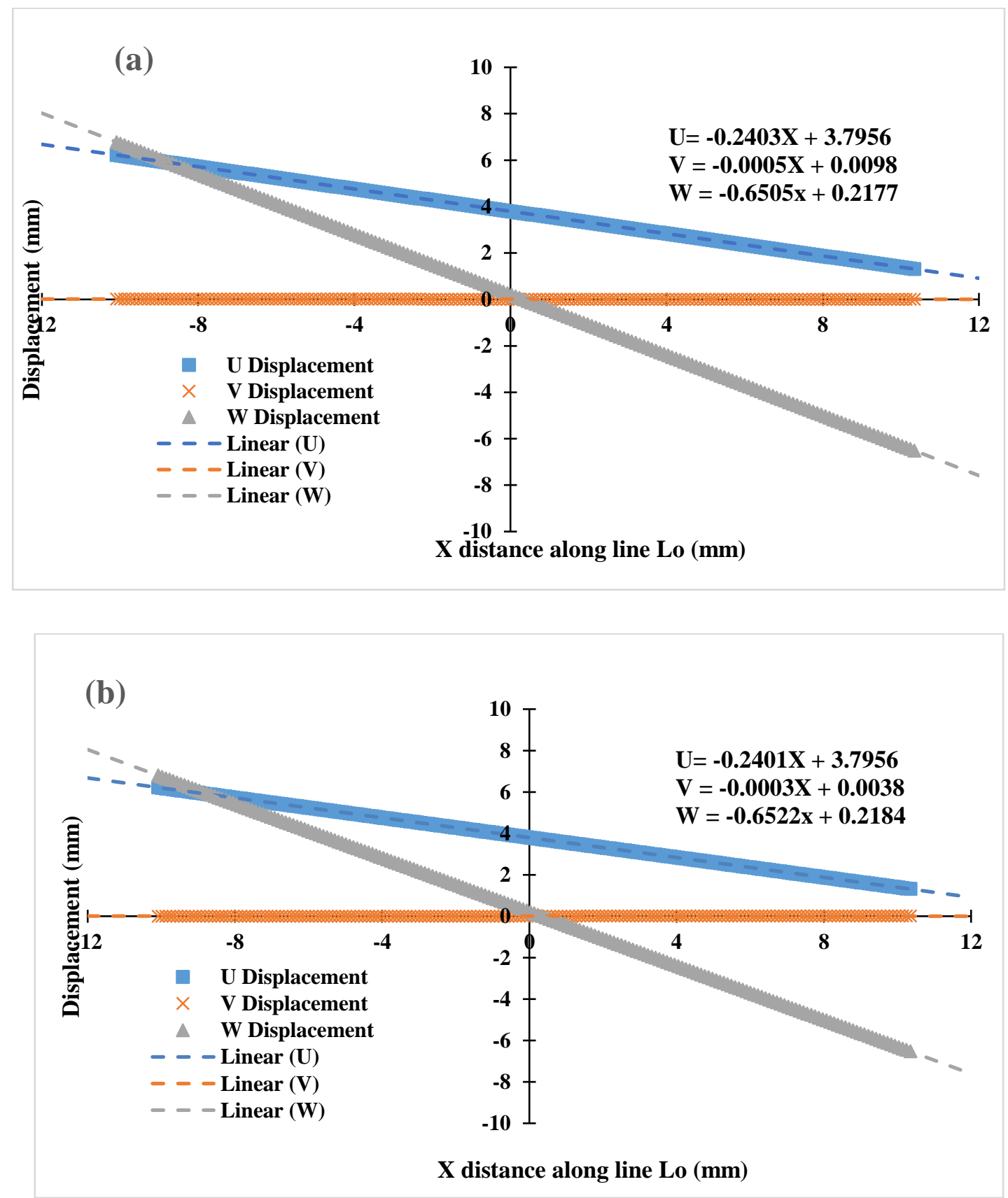

Figure 9 Typical $U, V, W$ displacement fields obtained using VIC-3D along line L0 for (a) LAC (b) SAC stereo pair for out-of-plane rotation $\theta=40^{\circ}$. 


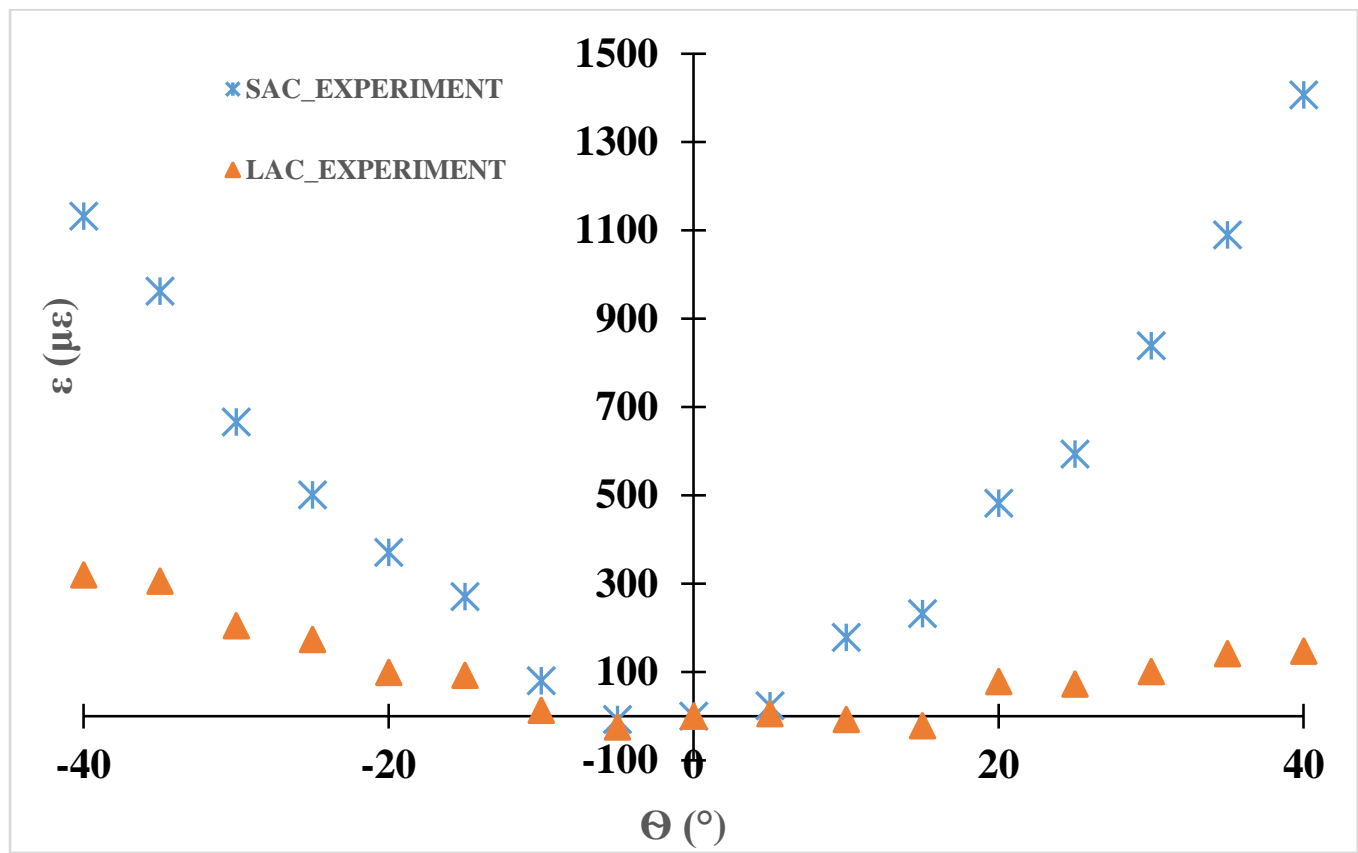

Figure 10 Average in-plane strain $\varepsilon_{x x}$ along line $\mathrm{L}_{0}$ as a function of out-of-plane rotation angle using SAC and LAC processes when specimen undergoes rigid body out-of-plane rotation.

\section{Analytical Predictions}

Since determining the exact camera parameters for our experiment is not possible, and yet the LAC parameters gave much lower strain error, in our simulations the LAC parameters are assumed to be the baseline set. Using these parameters in Table 2 and Table 3, the process to assess the effect of the SAC parameters is shown in the flow chart given in Figure 11. Details for the process outlined in Figure 11 are as follows. First, a line along $\mathrm{L}_{\mathrm{o}}$ in the object plane from $-10.5 \mathrm{~mm} \leq \mathrm{X} \leq 10.5 \mathrm{~mm}$ is defined. Secondly, the equation for the line when rotated out of plane about $\mathrm{X}=0$ by an arbitrary angle of rotation, $\theta$, is developed. Thirdly, the equation is evaluated at 85 uniformly spaced points with a spacing of $0.25 \mathrm{~mm}$ for each angle in the range $-40^{\circ} \leq \theta \leq 40^{\circ}$. The calculated locations are assumed to be the exact object positions. Fourthly, using Equ (2) with the LAC parameters, each point is projected into the sensor planes of camera 1 and camera 2 to define the "exact" sensor positions in both cameras. Fifthly, with the sensor positions in both stereo cameras known, the camera parameters in Equ (2) are replaced with SAC values and a least squares approach is employed with the four equations to define the corresponding threedimensional position in space. By performing this process for 85 points spaced $0.25 \mathrm{~mm}$ apart for each rotation angle, $\theta$, the displacements of each point are determined for each angle $\theta$ using the SAC parameters. Finally, the predicted 3D locations are used to determine the average strain $\varepsilon_{\mathrm{xx}}$ along the line for each angle $\theta$.

Since LAC parameters are used as the baseline set of parameters, the strain error results from the process defined above is compared to the difference in strains $\varepsilon_{\mathrm{xx}}$ obtained by SAC and LAC (see Figure 10). Figure 12 shows a direct comparison of 
the out-of-plane strain errors obtained using experimental measurements to those obtained using the independent theoretical formulation. Inspection of Figure 12 shows very good agreement between the two results, with a slight rotation of the SACLAC theoretical data relative to the experimental results. The results are believed to be due to the use of the LAC parameters as the baseline, since careful inspection of the measurements in Fig. 10 shows a slight bias in the LAC data with respect to rotation angle.

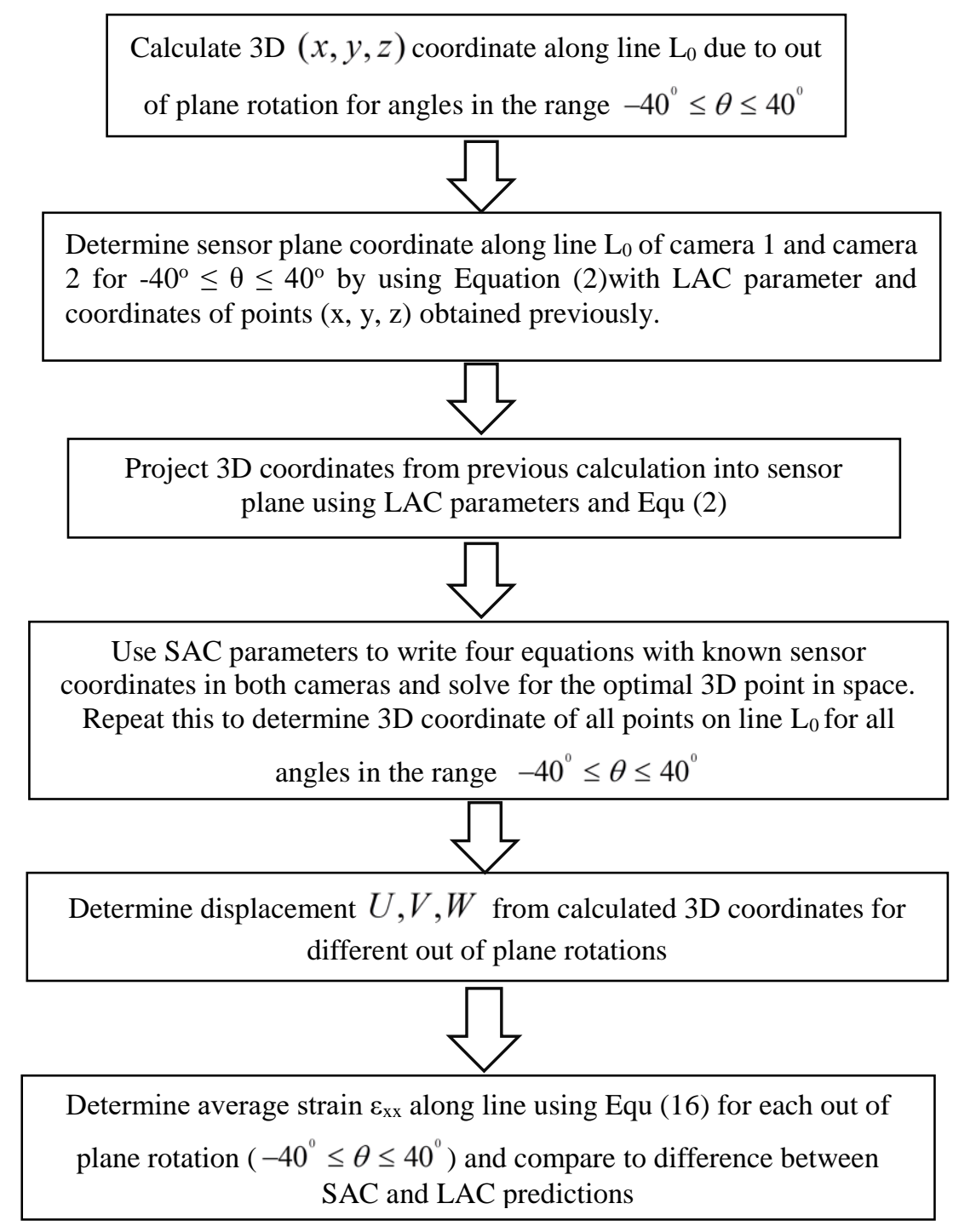

Figure 11 Flow chart for analytic strain $\varepsilon_{\mathrm{xx}}$ calculation from LAC To SAC projection 


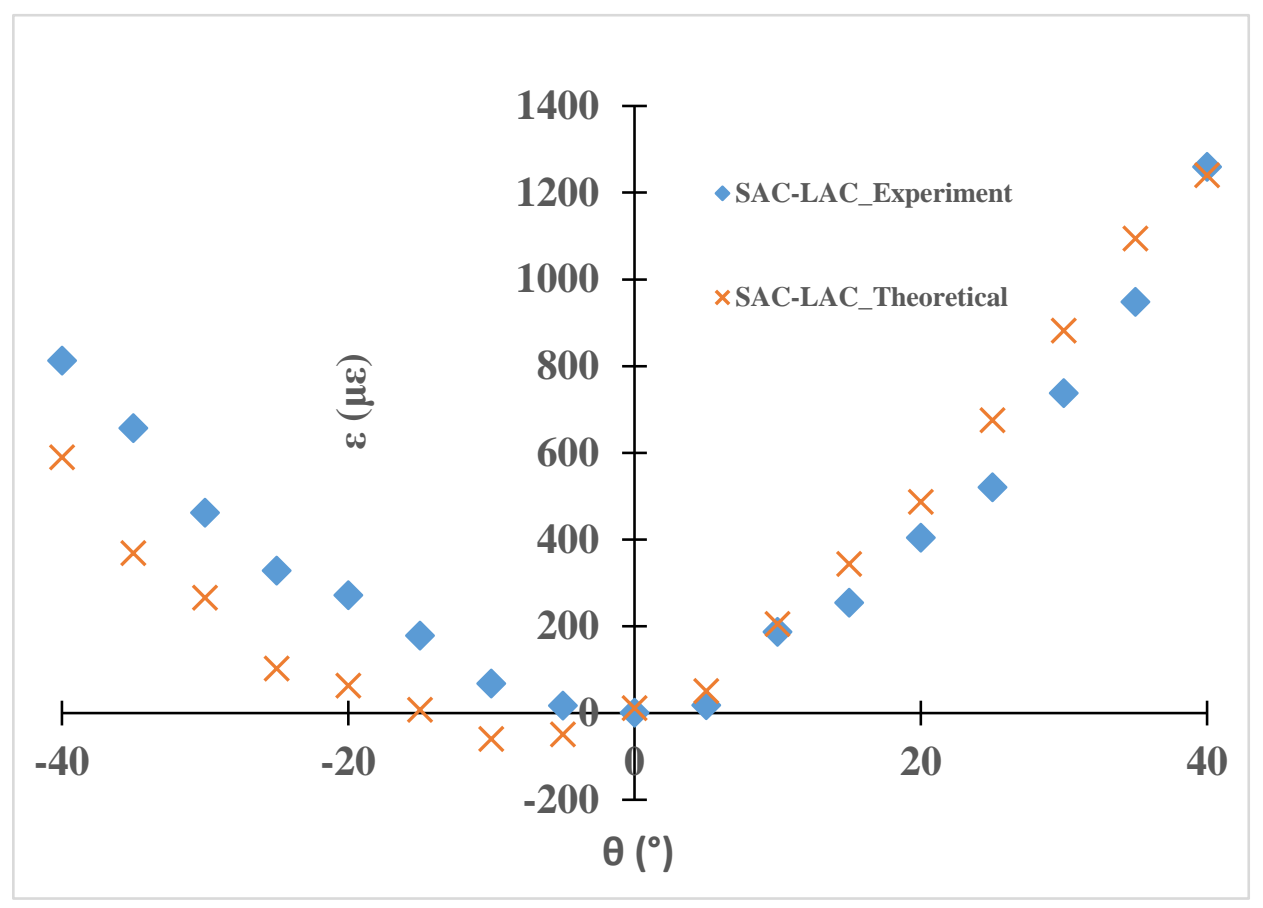

Figure 12 The experimental difference of strain $\varepsilon_{\mathrm{xx}}$ (SAC-LAC) and theoretical strain using SAC parameter projected from baseline sensor plane coordinates (LAC).

\section{Simulated images}

An FE-based image generator, earlier presented in [24] and used for stereo-DIC uncertainty quantification [25], is used in this section to further validate the results. Both synthetic calibration and test images are generated for the earlier described setup and these are analyzed with SAC and LAC calibration sets that are obtained from simulated images. An advantage of this approach is that the exact calibration parameters are known through the simulation-process.

Following the experimental procedure described previously, an SAC calibration image set of 61 images is generated in which a 12x9 calibration target with a spacing of $4 \mathrm{~mm}$ is translated over the entire field of view with rotation angles that are randomly generated within $\pm 10^{\circ}$ of out-of-plane rotation. An additional image set of 81 images for the LAC calibration is generated, in which the target is rotated with fixed intervals between $-40^{\circ}$ and $40^{\circ}$, so the calibration indicated in Section 4 could be replicated. Table 6 indicates the obtained internal calibration parameters, in which PC stands for "perfect calibration", i.e., the true calibration parameters as exported by the simulator. It is clear that the obtained calibration parameters from the LAC data set are closer to the true values compared to the calibration parameters from the SAC data set, which is caused by both the 
larger rotation angles of the calibration target, as well as the larger number of calibration images. The same trend can be seen in the external parameters in Table 7.

Table 6: Calibration parameters for simulated stereovision system

\begin{tabular}{|l|c|c|c|c|c|c|}
\hline Parameter & Cam 1-PC & Cam 2-PC & Cam 1-SAC & Cam 2-SAC & Cam 1-LAC & Cam 2-LAC \\
\hline$C_{x}$ (pixels) & 1376.000 & 1376.000 & 1351.545 & 1395.206 & 1369.169 & 1386.686 \\
\hline$C_{y}$ (pixels) & 1103.000 & 1103.000 & 1105.559 & 1101.207 & 1103.466 & 1101.794 \\
\hline$f \lambda_{x}$ (pixels) & 7180.000 & 7180.000 & 7223.176 & 7203.816 & 7183.369 & 7182.286 \\
\hline$f \lambda_{y}($ pixels) & 7180.000 & 7180.000 & 7217.520 & 7197.792 & 7176.988 & 7176.362 \\
\hline$\kappa_{1}\left(\right.$ pixels $\left.^{-2}\right)$ & -0.271 & -0.223 & -0.282 & -0.225 & -0.276 & -0.225 \\
\hline$\kappa_{2}\left(\right.$ pixels $\left.^{-4}\right)$ & 0.000 & 0.000 & 1.260 & 0.593 & 0.856 & 0.695 \\
\hline$\kappa_{6}\left(\right.$ pixels $\left.^{-6}\right)$ & 0.000 & 0.000 & -19.688 & -7.057 & -13.362 & -11.829 \\
& & & & & & \\
\hline
\end{tabular}

Table 7: Camera $1 \rightarrow 2$ transformation parameters for stereovision system

\begin{tabular}{|c|c|c|c|}
\hline Parameter & PC & SAC & LAC \\
\hline$\alpha\left(^{\circ}\right)$ & 0.000 & -0.0159 & -0.011 \\
\hline$\beta\left(^{\circ}\right)$ & -16.000 & -16.290 & -16.125 \\
\hline$\gamma\left({ }^{\circ}\right)$ & 0.000 & 0.002 & 0.002 \\
\hline $\mathrm{T}_{\mathrm{x}}(\mathrm{mm})$ & 83.503 & 83.662 & 83.421 \\
\hline $\mathrm{T}_{\mathrm{y}}(\mathrm{mm})$ & 0.000 & 0.102 & 0.017 \\
\hline $\mathrm{T}_{\mathrm{z}}(\mathrm{mm})$ & 11.736 & 11.130 & 11.816 \\
\hline Baseline $(\mathrm{mm})$ & 84.324 & 84.399 & 84.254 \\
& & & \\
\hline
\end{tabular}


A third image set, which replicates the rotation experiment (as indicated in Section 3), is generated with a standoff distance of $300 \mathrm{~mm}$ and a stereo angle of $16^{\circ}$. Images are generated for a specimen rotation between $-40^{\circ}$ and $40^{\circ}$, with steps of $5^{\circ}$. These images are then analyzed with another software platform [26] with the settings given in Table 8 and all three calibration sets. Please note that again 35mm lenses are used. However, the images are generated for a different camera type (AVT Manta), thus changing the $f \lambda$ factors.

Table 8: Parameters used for analysis of simulated images

\begin{tabular}{|c|c|}
\hline \multicolumn{2}{|c|}{ StereoDIC Analysis Parameters } \\
\hline Camera resolution (pixels) & $2752 \times 2206$ \\
\hline Subset size (pixels) & 19 \\
\hline Step size (pixels) & 7 \\
\hline Strain window & 5 \\
\hline Interpolation & B-spline \\
\hline Transformation & Affine \\
\hline
\end{tabular}

After analyzing the images with all three calibration datasets (i.e. PC, SAC and LAC) a horizontal line extraction is performed in the center region (as indicated in Figures 6 and 7) and the average in-plane strain $\varepsilon_{\mathrm{xx}}$ is plotted in Figure 13. The results obtained using the SAC calibration set shows the experimental and previous analytic parabolic behavior, with a maximum parasitic strain of $\approx 1450 \mu \varepsilon$. A lower bias is obtained when using the LAC calibration dataset, reducing the maximum strain bias to $\approx 500 \mu \varepsilon$, which is in very good correspondence with the experimental data. A third curve can now be added since the perfect calibration parameters are known and no strain bias is seen, as could be expected. Close correspondence to the experimental data is obtained as can be seen in Figure 14, which plots the SAC and LAC results for both the experimental images and the simulated images. 


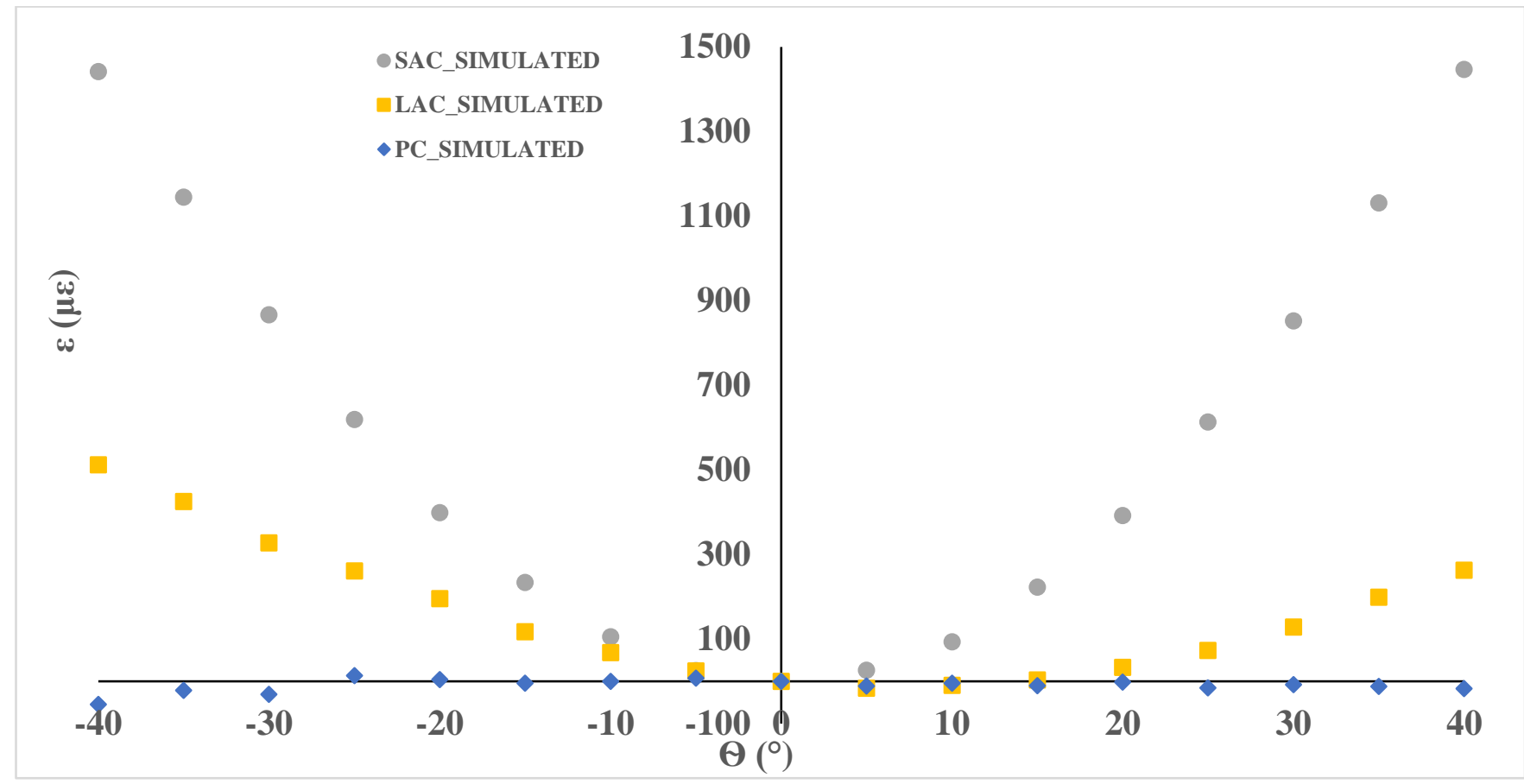

Figure 13 Average in-plane strain along the center line as a function of out-of-plane rotation angle using PC, SAC and LAC processes when specimen undergoes rigid body out-of-plane rotation.

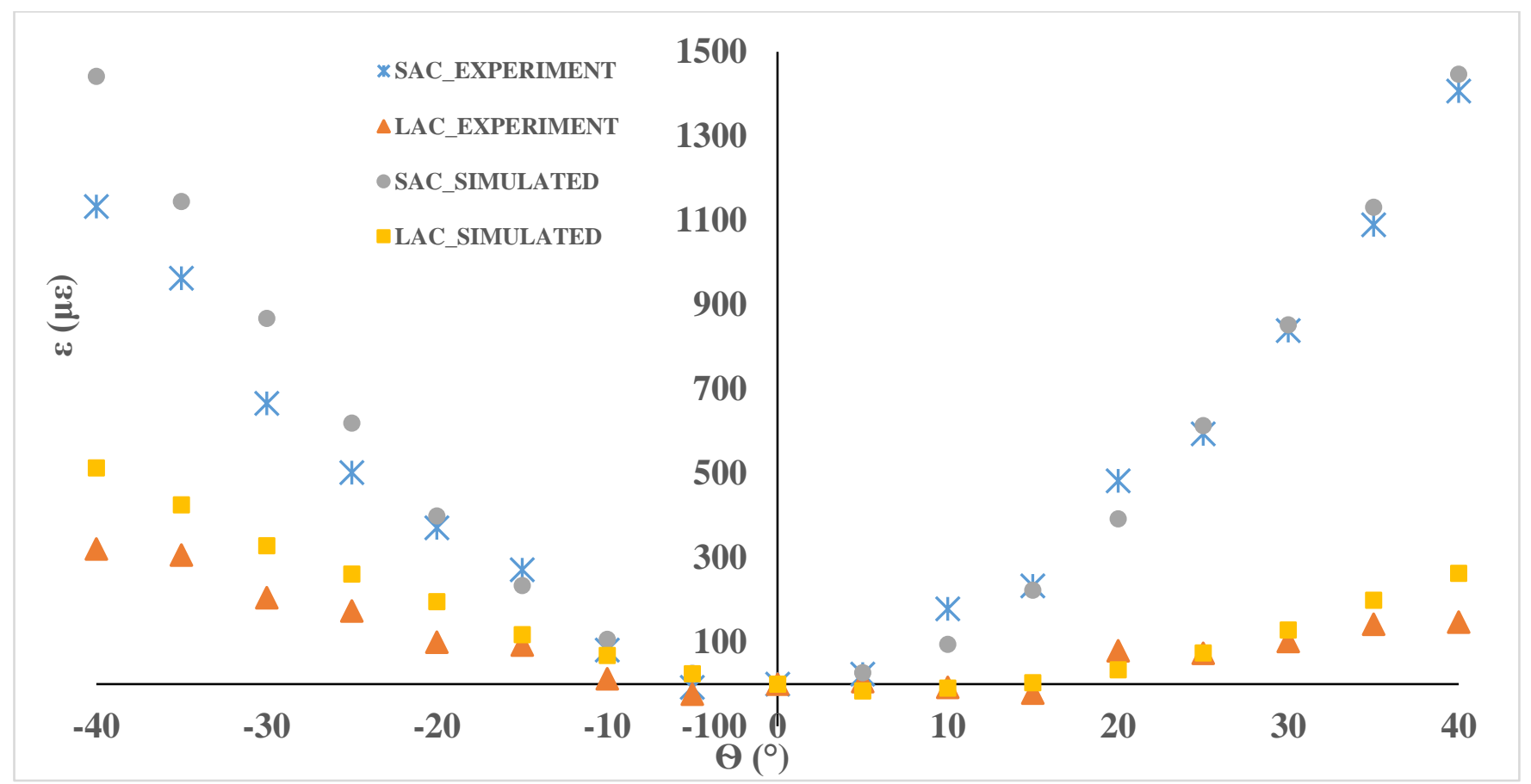

Figure 14 Comparison between experiment and simulated images. 


\section{Sensitivity Analysis}

Table 9 shows the LAC and SAC camera parameters (data range) obtained using bundle adjustment within a commercial image correlation software package [16]. To understand which parameters have the largest effect on strain measurement, the investigators performed variance based sensitivity analysis on Equ (16). Variance-based methods refer to ways of quantifying the contribution of each input parameter $\left(X_{i}\right)$ to the total variance of the output $(Y)$ results. The procedure employed to perform the sensitivity analysis follows the first four steps described in Section 6 to obtain the sensor plane coordinates when using the LAC camera parameters. As part of the analysis, independent intrinsic and extrinsic parameters are varied in the range spanned by both [LAC] - [SAC], and quasi-random Monte Carlo simulations is performed for N=1000 samples. The first order sensitivity coefficient and the total sensitivity coefficient are calculated using Equ (14) and Equ (15), respectively, for all input parameters. Figure 15 shows the result of sensitivity analysis from an evaluation of all independent intrinsic and extrinsic parameters for a rotation angle $\Theta=+40^{\circ}$. From Figure 15, it is seen that $\beta$ and $\alpha$ are the most dominant parameters for the model output variance. The $S_{\beta}=0.7755$ represents partial variance of parameter $\beta$ to total model output variability, while the

additive contribution of all input parameters gave $\sum_{i=1}^{16} S_{i}=0.9392$. This confirms that the first order sensitivity index of parameter, $\beta$, the camera pan angle, contribute $77.55 \%$. The parameter, $\alpha$, which is the tilt angle of each camera about the optic axis, contributes $16.37 \%$ of total model output variability. The sum of first order indices for the two most important parameters ( $\beta$ and $\alpha$ ) contribute $93.92 \%$ of the variability in the output which is equal to of total model variability $93.92 \%$ captured by 16 independent input parameters, as other input parameters have first order sensitivity indices less than $10^{-5}$. The interaction effect of parameters is calculated by $1-\sum_{i=1}^{16} S_{i}$.The total effect sensitivity index in Figure 15 shows that the $S_{T i}$ has a similar trend as $S_{i}$. That is, the ranking of parameters by $S_{i}$ values agreed well with those from $S_{T i}$ values. It is noted that all the $S_{T i}$ values are greater than $S_{i}$ values. This again suggests each parameter interacts as a pair, or with more parameters, to affect variability. $S_{T i}-S_{i}$ is used to estimate the interaction effect index of second order and/or higher order effects. The highest interaction effect is showed by $S_{T \beta}-S_{\beta}$, yielding $6.03 \%$. The parameter, $\alpha$, has an interaction effect of $5.87 \%$. The interaction effect shown by $S_{T \beta}-S_{\beta}$ suggests that this value is the result of interaction between $\beta$ and other parameters, but does not specifically indicate which other parameters are involved and what degree of interaction is present. 
The parameters $C_{x 1}, C_{x 2}, f \lambda_{x 1}, f \lambda_{x 2}, C_{y 1}, C_{y 2}, f \lambda_{y 1}, s_{1}, s_{2}, f \lambda_{y 2}, \gamma, T_{x}, T_{y}, T_{z}$ do not have a significant effect on model output as their $S_{i}$ and $S_{T i}$ values are very low compare to $S_{i}$ and $S_{T i}$ values of $\beta$ and $\alpha$ parameters. The low values of $S_{T i}$ of $C_{x 1}, C_{x 2}, f \lambda_{x 1}, f \lambda_{x 2}, C_{y 1}, C_{y 2}, f \lambda_{y 1}, s_{1}, s_{2}, f \lambda_{y 2}, \gamma, T_{x}, T_{y}, T_{z}$ also indicate no significant second-order interaction between the parameters.

Table 9 Data Range (LAC- SAC) for sensitivity analysis.

\begin{tabular}{|c|c|}
\hline Parameters & $\begin{array}{l}\text { Data Range } \\
\text { (LAC-SAC) }\end{array}$ \\
\hline$C_{x 1}$ (pixel) & $1656.108-1676.743$ \\
\hline$C_{y 1}$ (pixel) & $1359.802-1372.491$ \\
\hline$f \lambda_{x 1}$ (pixel) & $11140.06-11176.32$ \\
\hline$f \lambda_{y 1}$ (pixel) & $11138.96-11175.22$ \\
\hline Skew1 & $-1.635--3.742$ \\
\hline$C_{x 2}$ (pixel) & $1728.664-1712.878$ \\
\hline$C_{y 2}$ (pixel) & $1430.964-1432.081$ \\
\hline$f \lambda_{x 2}$ (pixel) & $11114.13-11134.51$ \\
\hline$f \lambda_{y 2}$ (pixel) & $11112.59-11133.54$ \\
\hline Skew2 & $0.958-2.538$ \\
\hline$\alpha_{\left({ }^{\circ}\right)}$ & $0.341-0.288$ \\
\hline$\beta_{\left({ }^{\circ}\right)}$ & $15.815-15.966$ \\
\hline$\gamma_{\left({ }^{\circ}\right)}$ & $1.482-1.489$ \\
\hline$T_{x}(\mathrm{~mm})$ & $-77.412--77.473$ \\
\hline$T_{y}(\mathrm{~mm})$ & $-1.006--0.992$ \\
\hline$T_{z}(\mathrm{~mm})$ & $10.487-10.229$ \\
\hline
\end{tabular}




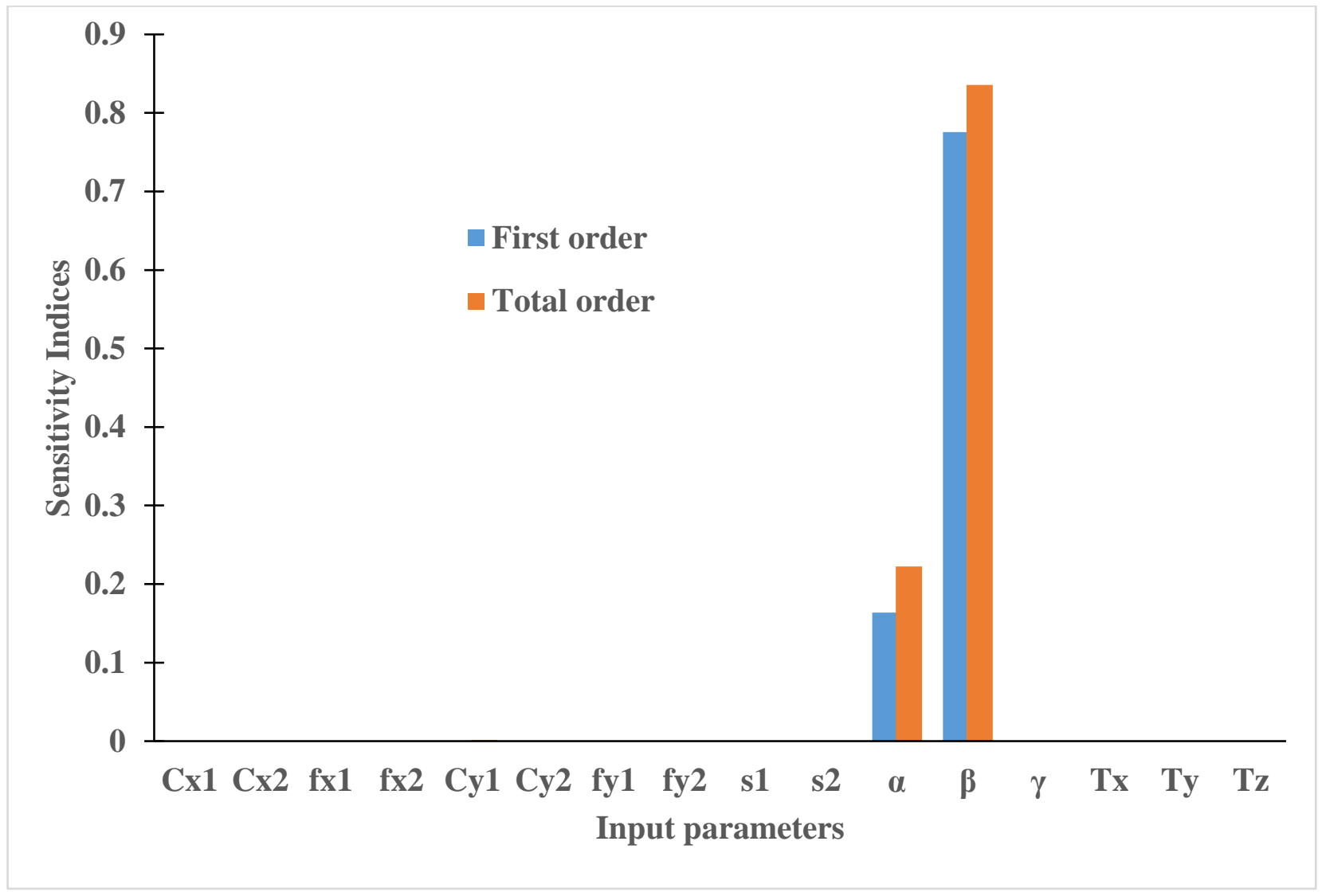

Figure 15 First order and Total order sensitivity indices of calibration parameters.

\section{Discussion of Analytical and Image Simulation Results}

The results in Figure 10, 12,13 and 14 show that the calibration process is sufficient to account for errors in strain $\varepsilon_{\mathrm{xx}}$ during rigid body out of plane rotation. From the sensitivity results, it is evident that the camera parameters $\beta$ and $\alpha$ are most important to accurately determine $\varepsilon_{x x}$. Thus, strain measurement errors are directly associated with differences in these parameter estimates for the SAC and LAC calibration processes. Though Table 2 and Table 3show that there is minimal variability in the parameters $\alpha, \beta, \gamma, T_{x}, T_{y}, T_{z}$, and somewhat higher variability for $C_{x 1}, C_{x 2}, f \lambda_{x 1}, f \lambda_{x 2}, s$, it is important to emphasize that the prediction of three-dimensional positions only depends upon the mean values determined during calibration for all parameters. Thus, the optimal SAC camera parameters are "biased" slightly due to the absence of sufficient out-of-plane motion in the images.

Regarding reasons for the observed differences in the key cameras parameters for LAC and SAC calibration processes, there are several points to note. First, each orientation of the calibration grid shown in Figure 3 adds 108 additional data points for each of the 81 target positions. Though this may be considered a lot of points, it is important to realize that the 22 camera 
parameters plus six additional extrinsic parameters per view must be obtained when performing bundle adjustment calibration. Thus, for SAC where there is no data available for large out of plane motion, the relatively small number of target views and target points are likely insufficient to accurately predict calibration parameters. Secondly, for LAC where there are target views that have out-of-plane motions spanning the range occurring in the experiments, the additional information is shown to substantially improve the accuracy of the optimal camera parameters, especially those most important in determining the Zcoordinate of points.

Based on these observations, the following recommendations for calibration are highlighted.

- When using standard calibration targets with limited points, using rotations and translations that span the entire range expected in an application will significantly improve the optimized camera parameter estimates.

- Increasing the number of calibration grid dots for each view so that more data is available to improve camera estimates is worthwhile, especially when calibration views may not span the range of motions that occur during an experiment.

- When the number of grid dots is limited, it may be worthwhile to consider using a speckle pattern for the calibration process. Such a pattern can be used to obtain thousands of data points at each rotation angle. Clearly, this would have a significant impact on reducing parameter variability and increasing the likelihood that optimal camera parameters are accurate while not increasing the number of additional extrinsic parameters that are needed to orient new views.

\section{Conclusions}

The influence of out-of-plane rotation in the range $-40^{\circ} \leq \theta \leq 40^{\circ}$ on strain measurements is investigated. The results illustrate that careful attention must be paid to the calibration process used for StereoDIC systems. Using only small rotations of the calibration target when the object undergoes much larger rigid body motions, it is shown that errors in strain in excess of $1,400 \mu \varepsilon$ are obtained. Using large target rotations that span the range of motions incurred by a target, it is also shown that strain errors can be suppressed to $<300 \mu \varepsilon$ in the experiments. Two separate approaches are used to determine whether the source of the errors is the calibration process. In one approach, calibration images for both the SAC and LAC processes are generated, as well as test images in order to further validate the proposed theory. In a separate approach, analytic results are obtained using both the experimental SAC and LAC parameters. Results from both approaches confirm that changing the calibration parameters is sufficient to predict the experimentally observed error trends. It is important to note that the usage of different 
DIC codes also excludes bias within the code as a reason for the observed strain errors. Sensitivity analysis performed on the camera parameters indicates that most of the strain error obtained using the SAC process is due to inaccuracy in two camera parameters: $\beta$ and $\alpha$. The remainder of the parameters $C_{x 1}, C_{x 2}, f \lambda_{x 1}, f \lambda_{x 2}, C_{y 1}, C_{y 2}, f \lambda_{y 1}, s_{1}, s_{2}, f \lambda_{y 2}, \gamma, T_{x}, T_{y}, T_{z}$ do not significantly affect the $\varepsilon_{x x}$ measurements.

\section{Acknowledgements}

The authors are grateful for the financial support from SPARC Graduate Research Grant Program, University of South Carolina, under grant numbers \# 15540-17-43825 and the Department of Mechanical Engineering, University of South Carolina, through a Teaching Assistantship.

\section{References}

[1] Schreier, H. W., Orteu, J. J., \& Sutton, M. A. (2009). Image correlation for shape, motion and deformation measurements. Springer US.

[2] Sutton, M. A., Wolters, W. J., Peters, W. H., Ranson, W. F., \& McNeill, S. R. (1983). Determination of displacements using an improved digital correlation method. Image and vision computing, 1(3), 133-139.

[3] Kahn-Jetter, Z. L., \& Chu, T. C. (1990). Three-dimensional displacement measurements using digital image correlation and photogrammic analysis. Experimental Mechanics, 30(1), 10-16.

[4] Luo, P. F., Chao, Y. J., Sutton, M. A., \& Peters Iii, W. H. (1993). Accurate measurement of three-dimensional deformations in deformable and rigid bodies using computer vision. Experimental Mechanics, 33(2), $123-132$.

[5] Luo, P. F., Chao, Y. J., \& Sutton, M. A. (1994). Application of stereo vision to three-dimensional deformation analyses in fracture experiments. Optical Engineering, 33(3), 981-990.

[6] Sutton, M. A., Yan, J. H., Tiwari, V., Schreier, H. W., \& Orteu, J. J. (2008). The effect of out-of-plane motion on 2D and 3D digital image correlation measurements. Optics and Lasers in Engineering, 46(10), 746-757.

[7] Tiwari, V., Sutton, M. A., \& McNeill, S. R. (2007). Assessment of high speed imaging systems for 2D and 3D deformation measurements: methodology development and validation. Experimental mechanics, 47(4), 561-579.

[8] Yan, J. H., Sutton, M. A., Deng, X., \& Cheng, C. S. (2007). Mixed-mode fracture of ductile thin-sheet materials under combined in-plane and out-of-plane loading. International Journal of Fracture, 144(4), 297-321. 
[9] Sutton, M. A., Yan, J., Deng, X., Cheng, C. S., \& Zavattieri, P. (2007). Three-dimensional digital image correlation to quantify deformation and crack-opening displacement in ductile aluminum under mixed-mode I/III loading. Optical Engineering, 46(5), 051003-051003.

[10] O. Ravn, N. A. Andersen, and A. T. Sorensen. Auto-calibration in Automation Systems using Vision. In 3rd International Symposium on Experimental Robotics (ISER’93), pages 206-218, Japan, 1993.

[11] Triggs, B., McLauchlan, P. F., Hartley, R. I., \& Fitzgibbon, A. W. (1999, September). Bundle adjustment—a modern synthesis. In International workshop on vision algorithms (pp. 298-372). Springer Berlin Heidelberg.

[12] Hartley, R., \& Zisserman, A. (2003). Multiple view geometry in computer vision. Cambridge university press.

[13] Yasmeen, F., Rajan, S., Sutton, M. A., \& Schreier, H. W. (2017). Experimental Study of Measurement Errors in 3DDIC Due to Out-of-Plane Specimen Rotation. In International Digital Imaging Correlation Society (pp. 211-215). Springer, Cham.

[14] Sutton, M. A., Helm, J. D., \& Boone, M. L. (2001). Experimental study of crack growth in thin sheet 2024-T3 aluminum under tension-torsion loading. International journal of fracture, 109(3), 285-301.

[15] Shukla, A., \& Dally, J. W. (2010). Experimental solid mechanics (p. 668). Knoxville: College House Enterprises.

[16] VIC-3D, Correlated Solutions Incorporated, 121 Dutchman Blvd, Irmo, SC 29063. www.correlatedsolutions.com.

[17] Sutton, M. A., Digital image correlation. In W. N. Sharpe, Jr., editor, Springer Handbook of Experimental Solid Mechanics, Springer, Berlin, 2008. ISBN: 978-0-387-26883-5.

[18] Christopher Frey, H., \& Patil, S. R. (2002). Identification and review of sensitivity analysis methods. Risk analysis, $22(3), 553-578$.

[19] Salehi, F., Prasher, S. O., Amin, S., Madani, A., Jebelli, S. J., Ramaswamy, H. S., Tan, C, Drury, C.F \& Yang, C. C. (2000). Prediction of annual nitrate-N losses in drain outflows with artificial neural networks. Transactions of the ASAE, 43(5), 1137-1143.

[20] Andersson, F. O., Åberg, M., \& Jacobsson, S. P. (2000). Algorithmic approaches for studies of variable influence, contribution and selection in neural networks. Chemometrics and intelligent laboratory systems, 51(1), 61-72.

[21] Geldermann, J., \& Rentz, O. (2001). Integrated technique assessment with imprecise information as a support for the identification of best available techniques (BAT). OR-Spektrum, 23(1), 137-157. 
[22] Sobol', I. (1990). Sensitivity estimates for nonlinear mathematical models. Matematicheskoe Modelirovanie 2, 112118. in Russian, translated in English in Sobol' , I. (1993). Sensitivity analysis for nonlinear mathematical models. Mathematical Modeling \& Computational Experiment (Engl. Transl.), 1993, 1, 407-414.

[23] Saltelli, A., Annoni, P., Azzini, I., Campolongo, F., Ratto, M., \& Tarantola, S. (2010). Variance based sensitivity analysis of model output. Design and estimator for the total sensitivity index. Computer Physics Communications, 181(2), 259-270.

[24] Balcaen R., Wittevrongel L., Reu P.L., Lava P., Debruyne D. (2017). Stereo-DIC calibration and speckle image generator based on FE formulations, Experimental Mechanics, 57(5), 703-718.

[25] Balcaen R., Reu P.L., Lava P., Debruyne D. (2017). Stereo-DIC uncertainty quantification based on simulated images, Accepted in Experimental Mechanics.

[26] MatchID, MatchIDMBC, Wijmenstraat 21T, B-9030 Gent-Belgium. http://www.matchidmbc.com/. 